\title{
DOMAIN DECOMPOSITION ALGORITHMS FOR TIME-HARMONIC MAXWELL EQUATIONS WITH DAMPING
}

\author{
Ana Alonso Rodriguez ${ }^{1}$ and Alberto Valli ${ }^{2}$
}

\begin{abstract}
Three non-overlapping domain decomposition methods are proposed for the numerical approximation of time-harmonic Maxwell equations with damping (i.e., in a conductor). For each method convergence is proved and, for the discrete problem, the rate of convergence of the iterative algorithm is shown to be independent of the number of degrees of freedom.
\end{abstract}

Mathematics Subject Classification. 65N55, 65N30.

Received: March 23, 2000. Revised: March 30, 2001.

\section{INTRODUCTION}

The time-harmonic Maxwell equations are derived from the complete Maxwell equations assuming that both the electric field $\mathcal{E}$ and the magnetic field $\mathcal{H}$ are of the form $\mathcal{E}(t, \mathbf{x})=\operatorname{Re}[\mathbf{E}(\mathbf{x}) \exp (\mathrm{i} \omega t)], \mathcal{H}(t, \mathbf{x})=$ $\operatorname{Re}[\mathbf{H}(\mathbf{x}) \exp (\mathrm{i} \omega t)]$, where $\omega \neq 0$ is a given angular frequency. Let $\Omega \subset \mathbb{R}^{3}$ be a bounded Lipschitz polyhedron with unit outward normal $\mathbf{n}$. Let $\varepsilon(\mathbf{x}), \mu(\mathbf{x})$ and $\sigma(\mathbf{x})$ denote respectively the dielectric constant, the magnetic permeability and the conductivity of the medium. The time-harmonic Maxwell equations reads:

$$
\begin{cases}(\mathrm{i} \omega \varepsilon+\sigma) \mathbf{E}-\operatorname{rot} \mathbf{H}=\mathbf{J} & \text { in } \Omega \\ \mathrm{i} \omega \mu \mathbf{H}+\operatorname{rot} \mathbf{E}=\mathbf{0} & \text { in } \Omega\end{cases}
$$

where $\mathbf{J}=\mathbf{J}(\mathbf{x})$ is a known function specifying the applied current density. (See [9] for a complete presentation of time-harmonic Maxwell equations.)

In the general case of anisotropic inhomogeneous media the coefficients $\varepsilon, \mu$ and $\sigma$ are $3 \times 3$ symmetric real matrices with entries in $L^{\infty}(\Omega)$. The matrices $\varepsilon$ and $\mu$ are assumed to be uniformly positive definite in $\Omega$. (A matrix $\zeta(\mathbf{x})$ is uniformly positive definite in $\Omega$ if there exists a constant $\zeta_{*}>0$ such that $\sum_{l, m=1}^{3} \zeta_{l, m}(\mathbf{x}) \xi_{l} \bar{\xi}_{m} \geq$ $\zeta_{*}|\boldsymbol{\xi}|^{2}$ for almost all $\mathbf{x} \in \Omega$ and for all $\boldsymbol{\xi} \in \mathbb{C}^{3}$.) The conductivity $\sigma$ is an uniformly positive definite matrix in a conductor and it is equal to 0 in an insulator.

As $\mu$ is non-singular we may eliminate the magnetic field $\mathbf{H}$ in (1) to obtain

$$
\operatorname{rot}\left(\mu^{-1} \operatorname{rot} \mathbf{E}\right)-\omega^{2} \varepsilon \mathbf{E}+\mathrm{i} \omega \sigma \mathbf{E}=\mathrm{i} \omega \mathbf{J} .
$$

Keywords and phrases. Time-harmonic Maxwell equations, domain decomposition methods, edge finite elements.

1 Dipartimento di Matematica, Università degli Studi di Milano, via Saldini 50, 20133 Milano, Italy.

e-mail: Ana.Alonso@mat.unimi.it, alonso@science.unitn.it

2 Dipartimento di Matematica, Università degli Studi di Trento, 38050 Povo (Trento), Italy. e-mail: valli@science.unitn.it

(C) EDP Sciences, SMAI 2001 
We shall also assume that the boundary of $\Omega$ is a perfect conductor so that

$$
\mathbf{E} \times \mathbf{n}=\mathbf{0} \quad \text { on } \partial \Omega .
$$

Though this is not the most relevant physical situation, we have preferred to consider it for the sake of simplicity. A more realistic boundary value problem is presented in Section 5 , and the results we are going to prove for the case of a perfectly conducting boundary also hold in that situation.

To state the weak formulation of this boundary value problem (2-3) we introduce the spaces

$$
\begin{aligned}
& H(\operatorname{rot} ; \Omega):=\left\{\mathbf{v} \in\left(L^{2}(\Omega)\right)^{3} \mid \operatorname{rot} \mathbf{v} \in\left(L^{2}(\Omega)\right)^{3}\right\} \\
& H_{0}(\operatorname{rot} ; \Omega):=\left\{\mathbf{v} \in H(\operatorname{rot} ; \Omega) \mid(\mathbf{v} \times \mathbf{n})_{\mid \partial \Omega}=\mathbf{0}\right\},
\end{aligned}
$$

and we consider the following bilinear form in $H(\operatorname{rot} ; \Omega)$

$$
a(\mathbf{w}, \mathbf{v}):=\left(\mu^{-1} \operatorname{rot} \mathbf{w}, \operatorname{rot} \mathbf{v}\right)-\omega^{2}(\varepsilon \mathbf{w}, \mathbf{v})+\mathrm{i} \omega(\sigma \mathbf{w}, \mathbf{v}),
$$

where $(\cdot, \cdot)$ denotes the $\left(L^{2}(\Omega)\right)^{3}$-scalar product for complex-valued vector functions. Assuming $\mathbf{J} \in\left(L^{2}(\Omega)\right)^{3}$, and setting, for simplifying notation, $\mathbf{F}=\mathrm{i} \omega \mathbf{J}$, the weak formulation of the boundary value problem (2-3) reads:

$$
\left\{\begin{array}{l}
\text { Find } \mathbf{E} \in H_{0}(\operatorname{rot} ; \Omega): \\
a(\mathbf{E}, \mathbf{v})=(\mathbf{F}, \mathbf{v}) \quad \forall \mathbf{v} \in H_{0}(\operatorname{rot} ; \Omega) .
\end{array}\right.
$$

When $\sigma$ is uniformly positive definite (namely, we are considering a conductor $\Omega$ ), it is easily seen that the bilinear form $a(\cdot, \cdot)$ is continuous and coercive in $H(\operatorname{rot} ; \Omega)$ and, as a consequence of the Lax-Milgram lemma for complex Hilbert spaces, we can state the following theorem, which is also valid for unbounded domains (see, e.g., [18]):

Theorem 1.1. Let $\Omega$ be an arbitrary domain in $\mathbb{R}^{3}$. If $\mu, \varepsilon$ and $\sigma$ are symmetric real matrices with coefficients in $L^{\infty}(\Omega)$ and $\mu$ and $\sigma$ are uniformly positive definite in $\Omega$, then there exists a unique solution of (5).

For the numerical approximation of problem (5), the edge elements introduced in [21] and [22] can be used. In [20] the use of these finite elements has been considered for problem (5), either for the case where $\sigma$ is assumed uniformly positive definite, or for the case $\sigma \equiv 0$. In both cases an optimal order error estimate is proved.

In the last years some domain decomposition methods for the numerical solution of the time-harmonic Maxwell equations have been proposed (see $[15,16,24,25]$, see also [3] and [4]). In [16] an iterative nonoverlapping domain decomposition method with Robin type transmission condition is given for the equation (2) in an insulator ( i.e., $\sigma \equiv 0$ ), with non-reflecting boundary conditions. In [15] a new iteration scheme is introduced for the same problem modifying the interface conditions proposed in [16]. In [3] and [4] we study iterative domain decomposition methods for the low-frequency model (namely, the case where the term $-\omega^{2} \varepsilon \mathbf{E}$ is neglected), both for the case of a conductor and for the case of the coupling insulator-conductor (eddy-current problem).

In this paper we are interested in domain decomposition methods for the problem in conductor, i.e., the complete equation (2) is considered and $\sigma$ is assumed to be uniformly positive definite in $\Omega$.

As we have already remarked, the presence of the damping term i $\omega \sigma \mathbf{E}$ ensures that the problem is coercive, and this property will play a crucial role in the rest of our analysis.

However, we note that the bilinear form $a(\cdot, \cdot)$ is not Hermitian symmetric. Moreover, if we split it as $a(\mathbf{w}, \mathbf{v})=a_{R}(\mathbf{w}, \mathbf{v})+\mathrm{i} a_{I}(\mathbf{w}, \mathbf{v})$, with $a_{R}(\mathbf{w}, \mathbf{v}):=\left(\mu^{-1} \operatorname{rot} \mathbf{w}, \operatorname{rot} \mathbf{v}\right)-\omega^{2}(\varepsilon \mathbf{w}, \mathbf{v})$ and $a_{I}(\mathbf{w}, \mathbf{v}):=\omega(\sigma \mathbf{w}, \mathbf{v})$, though it is coercive, neither $a_{R}(\cdot, \cdot)$ nor $a_{I}(\cdot, \cdot)$ is so. In [3], we find the same situation with a relevant difference: there $a_{R}(\cdot, \cdot)$ is positive semidefinite, while in the present situation this property is not satisfied. Therefore, the arguments used in [3] to prove convergence of a Dirichlet/Neumann iteration for the low-frequency model do not work in the present case. 
We are going to see in the sequel that the main difficulty arises from the fact that the bilinear form $a(\cdot, \cdot)$ is not Hermitian symmetric. We recall that, for non-overlapping domain decomposition methods, an analysis of convergence (also concerning the independence of the convergence rate of the mesh size) is based in general on the assumption that the problem is Hermitian symmetric (indeed, most often on the assumption that the problem is real and symmetric).

In this paper we aim to present a complete convergence analysis when the problem is complex and not Hermitian symmetric. More precisely, we introduce and analyse two families of non-overlapping domain decomposition methods: $\gamma$-Dirichlet/Robin methods and $\gamma$-Robin/Robin methods. These domain decomposition procedures are both related to algorithms used for advection-diffusion equations. The first one is related to the $\gamma$-DR method introduced in [5] and the second one is related to the modified $\gamma$-Robin-Robin method studied in [6] (which is a generalization of the well-known Neumann/Neumann method [1,10]). We also present briefly a modified Neumann/Neumann method analogous to the one presented in [7] for advection-diffusion equations.

The paper is organized as follows. In Section 2 we give an equivalent formulation of problem (5) in term of a new bilinear form which turns out to be more useful for our purposes. We state also an equivalent two-domain formulation for both continuous and discrete problems. In Section 3 we consider a general class of problems including those introduced in Section 2, and we present the non-overlapping domain decomposition methods that we shall analyse: $\gamma$-Dirichlet/Robin, $\gamma$-Robin/Robin and modified Neumann/Neumann. In Section 4 we study the convergence of the methods introduced in Section 3. In Section 5 all the methods are applied to the discrete time-harmonic Maxwell problem and, briefly, to other problems. Finally, in Section 6 we present some numerical tests concerning the $\gamma$-Dirichlet/Robin method: we show its efficiency and robustness, especially for the case $\gamma=0$ (the simplest one, as for that choice the algorithm reduces to the well-known Dirichlet/Neumann iterative scheme).

\section{Equivalent formulation of the problem}

As we already noticed, the bilinear form associated to the time-harmonic Maxwell problem can be written as $a(\cdot, \cdot)=a_{R}(\cdot, \cdot)+\mathrm{i} a_{I}(\cdot, \cdot)$. Due to the assumption that $\varepsilon, \mu$ and $\sigma$ are symmetric real matrices with entries in $L^{\infty}(\Omega)$ and uniformly positive definite, both $a_{R}(\cdot, \cdot)$ and $a_{I}(\cdot, \cdot)$ are Hermitian symmetric, continuous bilinear forms in $H(\operatorname{rot} ; \Omega)$; neither $a_{R}(\cdot, \cdot)$ nor $a_{I}(\cdot, \cdot)$ is coercive in $H(\operatorname{rot} ; \Omega)$. However, we can multiply the equation by a complex number $A-\mathrm{i} B$ in order to arrive to a new bilinear form $b(\cdot, \cdot)$ which can be split as $b(\cdot, \cdot)=$ $b_{R}(\cdot, \cdot)+\mathrm{i} b_{I}(\cdot, \cdot)$, with $b_{R}(\cdot, \cdot)$ Hermitian symmetric, continuous and coercive, and $b_{I}(\cdot, \cdot)$ Hermitian symmetric and continuous. Hence, $b(\cdot, \cdot)$ is real coercive, i.e., there exists a positive constant $\alpha_{R}$ such that $R e[b(\mathbf{v}, \mathbf{v})] \geq$ $\alpha_{R}\|\mathbf{v}\|_{H(\operatorname{rot} ; \Omega)}^{2}$ for each $\mathbf{v} \in H(\operatorname{rot} ; \Omega)$. We notice that this property is one of the assumptions we need for proving the convergence results in Section 4 (see Ths. 4.2 and 4.4); at this level, the positivity assumption on $\sigma$ (i.e., the damped character of the problem) turns out to be crucial.

Let $b(\cdot, \cdot)$ be defined as

$$
\begin{aligned}
b(\mathbf{w}, \mathbf{v}) & :=(A-\mathrm{i} B) a(\mathbf{w}, \mathbf{v}) \\
& =\int_{\Omega}\left[A \mu^{-1} \operatorname{rot} \mathbf{w} \cdot \operatorname{rot} \overline{\mathbf{v}}+\left(B \omega \sigma-A \omega^{2} \varepsilon\right) \mathbf{w} \cdot \overline{\mathbf{v}}\right]+\mathrm{i} \int_{\Omega}\left[\left(A \omega \sigma+B \omega^{2} \varepsilon\right) \mathbf{w} \cdot \overline{\mathbf{v}}-B \mu^{-1} \operatorname{rot} \mathbf{w} \cdot \operatorname{rot} \overline{\mathbf{v}}\right] \\
& =b_{R}(\mathbf{w}, \mathbf{v})+\mathrm{i} b_{I}(\mathbf{w}, \mathbf{v}) .
\end{aligned}
$$

The bilinear forms $b_{R}(\cdot, \cdot)$ and $b_{I}(\cdot, \cdot)$ are clearly Hermitian symmetric and continuous in $H($ rot; $\Omega$ ). Moreover we have

Proposition 2.1. If $\mu^{-1}, \varepsilon$ and $\sigma$ are symmetric matrices with entries in $L^{\infty}(\Omega)$ and $\mu^{-1}, \sigma$ are uniformly positive definite in $\Omega$, then there exists a positive constant $K$ such that for each $A>0$ and $B \omega>K$ the bilinear form

is coercive in $H(\operatorname{rot} ; \Omega)$.

$$
b_{R}(\mathbf{w}, \mathbf{v}):=\int_{\Omega}\left[A \mu^{-1} \operatorname{rot} \mathbf{w} \cdot \operatorname{rot} \overline{\mathbf{v}}+\left(B \omega \sigma-A \omega^{2} \varepsilon\right) \mathbf{w} \cdot \overline{\mathbf{v}}\right]
$$


Proof. Since $\mu^{-1}$ is uniformly positive definite in $\Omega$ it follows that $A \mu^{-1}$ is uniformly positive definite for each $A>0$. Moreover, since $\sigma$ is uniformly positive definite in $\Omega$ and $\varepsilon$ has entries in $L^{\infty}(\Omega)$, there exists positive constants $\sigma_{*}$ and $\varepsilon^{*}$ such that

$$
\begin{aligned}
& \sigma(\mathbf{x}) \boldsymbol{\xi} \cdot \boldsymbol{\xi} \geq \sigma_{*}|\boldsymbol{\xi}|^{2}, \\
& \varepsilon(\mathbf{x}) \boldsymbol{\xi} \cdot \boldsymbol{\xi} \leq \varepsilon^{*}|\boldsymbol{\xi}|^{2},
\end{aligned}
$$

for almost all $\mathbf{x} \in \Omega$ and for all $\boldsymbol{\xi} \in \mathbb{C}^{3}$. Since $A>0$ and assuming $B \omega>0$ we have

$$
\left[\left(B \omega \sigma-A \omega^{2} \varepsilon\right) \boldsymbol{\xi}\right] \cdot \boldsymbol{\xi}=B \omega \sigma \boldsymbol{\xi} \cdot \boldsymbol{\xi}-A \omega^{2} \varepsilon \boldsymbol{\xi} \cdot \boldsymbol{\xi} \geq\left(B \omega \sigma_{*}-A \omega^{2} \varepsilon^{*}\right)|\boldsymbol{\xi}|^{2} .
$$

Hence, choosing

$$
A>0 \text { and } B \omega>A \frac{\omega^{2} \varepsilon^{*}}{\sigma_{*}}=: K>0,
$$

the bilinear form $b_{R}(\cdot, \cdot)$ is coercive in $H(\operatorname{rot} ; \Omega)$.

For simplicity in the sequel we take $A=1$. Defining $\mathbf{G}:=(1-i B) \mathbf{F}$ we have thus transformed the original problem (1) into

$$
\left\{\begin{array}{l}
\text { Find } \mathbf{E} \in H_{0}(\operatorname{rot} ; \Omega): \\
b(\mathbf{E}, \mathbf{v})=(\mathbf{G}, \mathbf{v}) \quad \forall \mathbf{v} \in H_{0}(\operatorname{rot} ; \Omega)
\end{array}\right.
$$

\section{Two-domain formulation}

Let the bounded domain $\Omega$ be decomposed in two subdomains $\Omega_{1}$ and $\Omega_{2}$ such that both $\Omega_{1}$ and $\Omega_{2}$ are Lipschitz polyhedrons, $\bar{\Omega}=\overline{\Omega_{1}} \cup \overline{\Omega_{2}}$ and $\Omega_{1} \cap \Omega_{2}=\emptyset$. We will set $\Gamma:=\overline{\Omega_{1}} \cap \overline{\Omega_{2}}$. On $\Gamma$ we consider $\mathbf{n}_{\Gamma}$, the unit outward normal vector of $\Omega_{1}$.

We introduce the spaces

the bilinear forms in $H\left(\operatorname{rot} ; \Omega_{j}\right)$

$$
V_{j}:=\left\{\mathbf{v}_{j} \in H\left(\operatorname{rot} ; \Omega_{j}\right) \mid\left(\mathbf{v}_{j} \times \mathbf{n}\right)_{\mid \partial \Omega_{j} \backslash \Gamma}=\mathbf{0}\right\},
$$

$$
b_{j}\left(\mathbf{w}_{j}, \mathbf{v}_{j}\right):=\int_{\Omega_{j}}\left[\mu^{-1} \operatorname{rot} \mathbf{w}_{j} \cdot \operatorname{rot} \overline{\mathbf{v}}_{j}+\left(B \omega \sigma-\omega^{2} \varepsilon\right) \mathbf{w}_{j} \cdot \overline{\mathbf{v}}_{j}\right]+\mathrm{i} \int_{\Omega_{j}}\left[\left(\omega \sigma+B \omega^{2} \varepsilon\right) \mathbf{w}_{j} \cdot \overline{\mathbf{v}}_{j}-B \mu^{-1} \operatorname{rot} \mathbf{w}_{j} \cdot \operatorname{rot} \overline{\mathbf{v}}_{j}\right]
$$

and the linear forms

for $j=1,2$.

$$
L_{j}\left(\mathbf{v}_{j}\right):=\int_{\Omega_{j}} \mathbf{G} \cdot \overline{\mathbf{v}}_{j}
$$

Set

$$
X_{\Gamma}:=\left\{\left[\mathbf{n}_{\Gamma} \times\left(\mathbf{v}_{1} \times \mathbf{n}_{\Gamma}\right)\right]_{\mid \Gamma}: \mathbf{v}_{1} \in V_{1}\right\}=\left\{\left[\mathbf{n}_{\Gamma} \times\left(\mathbf{v}_{2} \times \mathbf{n}_{\Gamma}\right)\right]_{\mid \Gamma}: \mathbf{v}_{2} \in V_{2}\right\},
$$

and let us introduce the trace operators

$$
\left\{\begin{array}{l}
\operatorname{Tr}_{j}: V_{j} \rightarrow X_{\Gamma} \\
\operatorname{Tr}_{j} \mathbf{v}_{j}:=\left[\mathbf{n}_{\Gamma} \times\left(\mathbf{v}_{j} \times \mathbf{n}_{\Gamma}\right)\right]_{\mid \Gamma} \quad \forall \mathbf{v}_{j} \in V_{j}
\end{array}\right.
$$

and extension operators

$$
\left\{\begin{array}{l}
\mathbf{R}_{j}: X_{\Gamma} \rightarrow V_{j} \\
\operatorname{Tr}_{j}\left(\mathbf{R}_{j} \boldsymbol{\xi}\right)=\boldsymbol{\xi} \quad \forall \boldsymbol{\xi} \in X_{\Gamma} .
\end{array}\right.
$$

The characterization of $X_{\Gamma}$ can be found in [14] for regular domains and in [12,13] for Lipschitz polyhedrons. It turns out that $X_{\Gamma}$ is a Hilbert space, intrinsically dependent on $\Gamma$ and not on $\Omega_{1}$ or $\Omega_{2}$, strictly contained in 
$\left(H^{-1 / 2}(\Gamma)\right)^{3}$, the dual space of $\left(H^{1 / 2}(\Gamma)\right)^{3}$. Just to give an idea, in the case $\Gamma \cap \partial \Omega=\emptyset$ the characterization result reads:

$$
X_{\Gamma}=\left\{\boldsymbol{\xi} \in\left(H^{-1 / 2}(\Gamma)\right)^{3} \mid \boldsymbol{\xi} \cdot \mathbf{n}=0 \text { and } \operatorname{rot}_{\tau} \boldsymbol{\xi} \in H^{-1 / 2}(\Gamma)\right\}
$$

For the construction of continuous extension operators $\mathbf{R}_{j}$ see [2]. There the following assumptions on the geometry of the domain $\Omega$ are required: $\partial \Omega$ is Lipschitz and $H(\operatorname{rot} ; \Omega) \cap H_{0}(\operatorname{div} ; \Omega) \subset\left(H^{1}(\Omega)\right)^{3}$. This last hypothesis is satisfied if $\partial \Omega \in C^{1,1}$ or $\Omega$ is a convex polyhedron. However, using the results in [12,13] and the same arguments of [2], it is possible to drop the hypothesis $H(\operatorname{rot} ; \Omega) \cap H_{0}(\operatorname{div} ; \Omega) \subset\left(H^{1}(\Omega)\right)^{3}$ and to construct a continuous extension operator in a Lipschitz polyhedron, not necessarily convex.

The two-domain formulation of problem (6) reads:

$$
\left\{\begin{array}{l}
\text { find }\left(\mathbf{E}_{1}, \mathbf{E}_{2}\right) \in V_{1} \times V_{2}: \\
b_{1}\left(\mathbf{E}_{1}, \mathbf{v}_{1}\right)=L_{1}\left(\mathbf{v}_{1}\right) \quad \forall \mathbf{v}_{1} \in H_{0}\left(\operatorname{rot} ; \Omega_{1}\right) \\
\operatorname{Tr}_{1} \mathbf{E}_{1}=\operatorname{Tr}_{2} \mathbf{E}_{2} \\
b_{2}\left(\mathbf{E}_{2}, \mathbf{v}_{2}\right)=L_{2}\left(\mathbf{v}_{2}\right) \quad \forall \mathbf{v}_{2} \in H_{0}\left(\operatorname{rot} ; \Omega_{2}\right) \\
b_{2}\left(\mathbf{E}_{2}, \mathbf{R}_{2} \boldsymbol{\xi}\right)=L_{2}\left(\mathbf{R}_{2} \boldsymbol{\xi}\right)+L_{1}\left(\mathbf{R}_{1} \boldsymbol{\xi}\right)-b_{1}\left(\mathbf{E}_{1}, \mathbf{R}_{1} \boldsymbol{\xi}\right) \quad \forall \boldsymbol{\xi} \in X_{\Gamma} .
\end{array}\right.
$$

The equivalence of the formulations (6) and (7) can be easily proved (see, for instance, Alonso and Valli [3], where a similar situation is considered).

For the numerical approximation we will use the edge elements introduced by Nédélec (see [21] and [22]). They are rot-conforming finite elements. We consider a family of triangulations $\left\{\mathcal{T}_{h}\right\}_{h>0}$ of $\Omega$ matching in the interface, i.e., we assume that each element of $\mathcal{T}_{h}$ only intersects either $\Omega_{1}$ or $\Omega_{2}$. In this way we construct finite dimensional spaces $V_{j, h} \subset V_{j}, V_{j, h}^{0}:=V_{j, h} \cap H_{0}\left(\operatorname{rot} ; \Omega_{j}\right)$ and on the interface we have the finite elements

$$
X_{\Gamma, h}:=\left\{\left[\mathbf{n}_{\Gamma} \times\left(\mathbf{v}_{1, h} \times \mathbf{n}_{\Gamma}\right)\right]_{\mid \Gamma} \mid \mathbf{v}_{1, h} \in V_{1, h}\right\}=\left\{\left[\mathbf{n}_{\Gamma} \times\left(\mathbf{v}_{2, h} \times \mathbf{n}_{\Gamma}\right)\right]_{\mid \Gamma} \mid \mathbf{v}_{2, h} \in V_{2, h}\right\} .
$$

The finite dimensional approximation problem reads:

$$
\left\{\begin{aligned}
\text { find }\left(\mathbf{E}_{1, h}, \mathbf{E}_{2, h}\right) \in V_{1, h} \times V_{2, h}: \\
b_{1}\left(\mathbf{E}_{1, h}, \mathbf{v}_{1, h}\right)=L_{1}\left(\mathbf{v}_{1, h}\right) \quad \forall \mathbf{v}_{1, h} \in V_{1, h}^{0} \\
\operatorname{Tr}_{1} \mathbf{E}_{1, h}=\operatorname{Tr}_{2} \mathbf{E}_{2, h} \\
b_{2}\left(\mathbf{E}_{2, h}, \mathbf{v}_{2, h}\right)=L_{2}\left(\mathbf{v}_{2, h}\right) \quad \forall \mathbf{v}_{2, h} \in V_{2, h}^{0} \\
b_{2}\left(\mathbf{E}_{2, h}, \mathbf{R}_{2, h} \boldsymbol{\xi}_{h}\right)=L_{2}\left(\mathbf{R}_{2, h} \boldsymbol{\xi}_{h}\right) \\
+L_{1}\left(\mathbf{R}_{1, h} \boldsymbol{\xi}_{h}\right)-b_{1}\left(\mathbf{E}_{1, h}, \mathbf{R}_{1, h} \boldsymbol{\xi}_{h}\right) \quad \forall \boldsymbol{\xi}_{h} \in X_{\Gamma, h},
\end{aligned}\right.
$$

where $\mathbf{R}_{j, h}$ is any extension operator from $X_{\Gamma, h}$ to $V_{j, h}, j=1,2$. 


\section{NON-OVERLAPPING DOMAIN DECOMPOSITION METHODS}

Now we consider an abstract problem whose structure is the same of problems (7) and (8):

$$
\left\{\begin{array}{l}
\text { find }\left(u_{1}, u_{2}\right) \in \mathcal{V}_{1} \times \mathcal{V}_{2}: \\
\mathcal{A}_{1}\left(u_{1}, v_{1}\right)=\mathcal{L}_{1}\left(v_{1}\right) \quad \forall v_{1} \in \mathcal{V}_{1}^{0} \\
\operatorname{Tr}_{1} u_{1}=\operatorname{Tr}_{2} u_{2} \\
\mathcal{A}_{2}\left(u_{2}, v_{2}\right)=\mathcal{L}_{2}\left(v_{2}\right) \quad \forall v_{2} \in \mathcal{V}_{2}^{0} \\
\mathcal{A}_{2}\left(u_{2}, \mathcal{R}_{2} \xi\right)=\mathcal{L}_{2}\left(\mathcal{R}_{2} \xi\right)+\mathcal{L}_{1}\left(\mathcal{R}_{1} \xi\right)-\mathcal{A}_{1}\left(u_{1}, \mathcal{R}_{1} \xi\right) \quad \forall \xi \in \mathcal{X},
\end{array}\right.
$$

where for $j=1,2, \mathcal{V}_{j}$ and $\mathcal{X}$ are complex Hilbert spaces, $\operatorname{Tr}_{j}: \mathcal{V}_{j} \rightarrow \mathcal{X}$ is a linear and continuous trace operator, $\mathcal{R}_{j}: \mathcal{X} \rightarrow \mathcal{V}_{j}$ is a linear and continuous extension operator (hence $\operatorname{Tr}_{j}\left(\mathcal{R}_{j} \xi\right)=\xi$ for all $\xi \in \mathcal{X}$ ), $\mathcal{V}_{j}^{0}:=\left\{v_{j} \in \mathcal{V}_{j}: \operatorname{Tr}_{j} v_{j}=0\right\}$ and $\mathcal{L}_{j}$ is a continuous linear form in $\mathcal{V}_{j} . \mathcal{A}_{j}$ is a bilinear form such that $\mathcal{A}_{j}(\cdot, \cdot)=\mathcal{A}_{R, j}(\cdot, \cdot)+i \mathcal{A}_{I, j}(\cdot, \cdot)$, where $\mathcal{A}_{R, j}$ and $\mathcal{A}_{I, j}$ are Hermitian and continuous bilinear forms and $\mathcal{A}_{R, j}$ is coercive.

In the sequel we will use the following notation: if $X$ is a complex Hilbert space and $X^{\prime}$ is its dual space, we denote by $(\cdot, \cdot)_{X}$ the inner product in $X$, by $\|\cdot\|_{X}$ the associated norm and by $\langle\cdot, \cdot\rangle$ the duality pairing between $X^{\prime}$ and $X$.

The two-domain problem (9) can be also formulated in term of the Steklov-Poincaré operators. For $j=1,2$, we consider the extension $\mathcal{E}_{j}: \mathcal{X} \rightarrow \mathcal{V}_{j}$ defined in the following way: for each $\xi \in \mathcal{X}, \mathcal{E}_{j} \xi$ is the unique solution of

$$
\left\{\begin{array}{l}
\mathcal{E}_{j} \xi \in \mathcal{V}_{j}: \\
\mathcal{A}_{j}\left(\mathcal{E}_{j} \xi, v_{j}\right)=0 \quad \forall v_{j} \in \mathcal{V}_{j}^{0} \\
\operatorname{Tr}_{j}\left(\mathcal{E}_{j} \xi\right)=\xi
\end{array}\right.
$$

Then the local Steklov-Poincaré operators $\mathcal{S}_{j}: \mathcal{X} \rightarrow \mathcal{X}^{\prime}$ is defined as follows:

$$
\left\langle\mathcal{S}_{j} \xi, \nu\right\rangle:=\mathcal{A}_{j}\left(\mathcal{E}_{j} \xi, \mathcal{E}_{j} \nu\right), \quad \forall \xi, \nu \in \mathcal{X},
$$

and we also set $\mathcal{S}=\mathcal{S}_{1}+\mathcal{S}_{2}$.

Let $\Psi_{j}$ be the solution of

Now we can define $\Upsilon \in \mathcal{X}^{\prime}$ as

$$
\left\{\begin{array}{l}
\Psi_{j} \in \mathcal{V}_{j}^{0}: \\
\mathcal{A}_{j}\left(\Psi_{j}, v_{j}\right)=\mathcal{L}_{j}\left(v_{j}\right) \quad \forall v_{j} \in \mathcal{V}_{j}^{0} .
\end{array}\right.
$$

$$
\langle\Upsilon, \xi\rangle:=\sum_{j=1}^{2}\left[\mathcal{L}_{j}\left(\mathcal{E}_{j} \xi\right)-\mathcal{A}_{j}\left(\Psi_{j}, \mathcal{E}_{j} \xi\right)\right] .
$$

It is clear that the solution of (9) can be written as

$$
u_{j}=\mathcal{E}_{j} \zeta+\Psi_{j}
$$

where

$$
\left(\mathcal{S}_{1}+\mathcal{S}_{2}\right) \zeta=\Upsilon
$$


We are going to construct the solution of (11) as the limit of the Richardson method with a preconditioner. The different algorithms we propose correspond to different preconditioners.

\subsection{The $\gamma$-Dirichlet/Robin method}

This is, in fact, a family of methods depending on a real parameter $\gamma$. At each step we have a boundary value problem in $\Omega_{1}$ with a Dirichlet condition on $\Gamma$ and a boundary value problem in $\Omega_{2}$ with Robin boundary condition on $\Gamma$. Given $\gamma \in \mathbb{R}, \gamma \geq 0$, we propose the following iteration: being given $\zeta^{0} \in \mathcal{X}$, for each $n \geq 0$ solve

$$
\left\{\begin{array}{l}
u_{1}^{n+1} \in \mathcal{V}_{1}: \\
\mathcal{A}_{1}\left(u_{1}^{n+1}, v_{1}\right)=\mathcal{L}_{1}\left(v_{1}\right) \quad \forall v_{1} \in \mathcal{V}_{1}^{0} \\
\operatorname{Tr}_{1} u_{1}^{n+1}=\zeta^{n},
\end{array}\right.
$$

then

$$
\left\{\begin{array}{l}
u_{2}^{n+1} \in \mathcal{V}_{2}: \\
\mathcal{A}_{2}\left(u_{2}^{n+1}, v_{2}\right)=\mathcal{L}_{2}\left(v_{2}\right) \quad \forall v_{2} \in \mathcal{V}_{2}^{0} \\
\mathcal{A}_{2}\left(u_{2}^{n+1}, \mathcal{R}_{2} \xi\right)+\gamma\left(\operatorname{Tr}_{2} u_{2}^{n+1}, \xi\right) \mathcal{X}=\mathcal{L}_{2}\left(\mathcal{R}_{2} \xi\right) \\
\quad+\mathcal{L}_{1}\left(\mathcal{R}_{1} \xi\right)-\mathcal{A}_{1}\left(u_{1}^{n+1}, \mathcal{R}_{1} \xi\right)+\gamma\left(\operatorname{Tr}_{1} u_{1}^{n+1}, \xi\right) \mathcal{X} \quad \forall \xi \in \mathcal{X},
\end{array}\right.
$$

finally set

$$
\zeta^{n+1}=(1-\theta) \zeta^{n}+\theta \operatorname{Tr}_{2} u_{2}^{n+1}
$$

If, for any $k \in \mathbb{R}$ we consider the operators $\mathcal{S}_{j}^{k}: \mathcal{X} \rightarrow \mathcal{X}^{\prime}$

$$
\left\langle\mathcal{S}_{j}^{k} \xi, \nu\right\rangle:=\left\langle\mathcal{S}_{j} \xi, \nu\right\rangle+k(\xi, \nu) \mathcal{X}
$$

$j=1,2$, it is easy to see that

$$
\mathcal{S}_{2}^{\gamma}\left(\operatorname{Tr}_{2} u_{2}^{n+1}\right)=\Upsilon-\mathcal{S}_{1}^{-\gamma} \zeta^{n}
$$

hence

$$
\begin{aligned}
\zeta^{n+1} & =(1-\theta) \zeta^{n}+\theta\left(\mathcal{S}_{2}^{\gamma}\right)^{-1}\left(\Upsilon-\mathcal{S}_{1}^{-\gamma} \zeta^{n}\right) \\
& =\zeta^{n}+\theta\left(\mathcal{S}_{2}^{\gamma}\right)^{-1}\left[\Upsilon-\left(\mathcal{S}_{1}^{-\gamma}+\mathcal{S}_{2}^{\gamma}\right) \zeta^{n}\right] \\
& =\zeta^{n}+\theta\left(\mathcal{S}_{2}^{\gamma}\right)^{-1}\left(\Upsilon-\mathcal{S} \zeta^{n}\right) .
\end{aligned}
$$

The iterative procedure (12-14) is therefore equivalent to the Richardson method for problem (11) using $\mathcal{S}_{2}^{\gamma}$ as preconditioner. The iteration operator is

$$
\mathcal{T}_{\theta}=I-\theta\left(\mathcal{S}_{2}^{\gamma}\right)^{-1} \mathcal{S}
$$

\subsection{The $\gamma$-Robin/Robin method}

We consider another family of methods, depending on a real parameter $\gamma \geq 0$, that will be called $\gamma$ Robin/Robin (a generalization of the Neumann/Neumann method [1,10]): being given $\zeta^{0} \in \mathcal{X}$ for each $n \geq 0$ 
solve for $j=1,2$

$$
\left\{\begin{array}{l}
u_{j}^{n+1} \in \mathcal{V}_{j}: \\
\mathcal{A}_{j}\left(u_{j}^{n+1}, v_{j}\right)=\mathcal{L}_{j}\left(v_{j}\right) \quad \forall v_{j} \in \mathcal{V}_{j}^{0} \\
\operatorname{Tr}_{j} u_{j}^{n+1}=\zeta^{n}
\end{array}\right.
$$

then

$$
\left\{\begin{array}{l}
\Phi_{j}^{n+1} \in \mathcal{V}_{j}: \\
\mathcal{A}_{j}\left(\Phi_{j}^{n+1}, v_{j}\right)=0 \quad \forall v_{j} \in \mathcal{V}_{j}^{0} \\
\mathcal{A}_{j}\left(\Phi_{j}^{n+1}, \mathcal{R}_{j} \xi\right)+\gamma\left(\operatorname{Tr}_{j} \Phi_{j}^{n+1}, \xi\right) \mathcal{X}=\mathcal{L}_{1}\left(\mathcal{R}_{1} \xi\right)-\mathcal{A}_{1}\left(u_{1}^{n+1}, \mathcal{R}_{1} \xi\right) \\
\quad+\mathcal{L}_{2}\left(\mathcal{R}_{2} \xi\right)-\mathcal{A}_{2}\left(u_{2}^{n+1}, \mathcal{R}_{2} \xi\right) \quad \forall \xi \in \mathcal{X},
\end{array}\right.
$$

finally set

$$
\zeta^{n+1}=\zeta^{n}+\theta\left(\operatorname{Tr}_{1} \Phi_{1}^{n+1}+\operatorname{Tr}_{2} \Phi_{2}^{n+1}\right) .
$$

In order to write this iteration as a preconditioned Richardson method for the Steklov-Poincaré problem (11), it is easy to see that

$$
\mathcal{S}_{j}^{\gamma}\left(\operatorname{Tr}_{j} \Phi_{j}^{n+1}\right)=\Upsilon-\left(\mathcal{S}_{1}+\mathcal{S}_{2}\right) \zeta^{n}, \quad j=1,2,
$$

so

$$
\zeta^{n+1}=\zeta^{n}+\theta\left[\left(\mathcal{S}_{1}^{\gamma}\right)^{-1}+\left(\mathcal{S}_{2}^{\gamma}\right)^{-1}\right]\left(\Upsilon-\mathcal{S} \zeta^{n}\right) .
$$

Then the iterative procedure (15-17) is equivalent to the Richardson method for problem (11) using $\left[\left(\mathcal{S}_{1}^{\gamma}\right)^{-1}+\right.$ $\left.\left(\mathcal{S}_{2}^{\gamma}\right)^{-1}\right]^{-1}$ as a preconditioner. In particular the iteration operator in (17) is given by

$$
\widetilde{\mathcal{T}}_{\theta}=I-\theta\left[\left(\mathcal{S}_{1}^{\gamma}\right)^{-1}+\left(\mathcal{S}_{2}^{\gamma}\right)^{-1}\right] \mathcal{S} .
$$

\subsection{The modified Neumann/Neumann method}

Another domain decomposition method that we can consider is the following modification of Neumann/Neumann method: being given $\zeta^{0} \in \mathcal{X}$ for each $n \geq 0$ solve for $j=1,2$

$$
\left\{\begin{array}{l}
u_{j}^{n+1} \in \mathcal{V}_{j}: \\
\mathcal{A}_{j}\left(u_{j}^{n+1}, v_{j}\right)=\mathcal{L}_{j}\left(v_{j}\right) \quad \forall v_{j} \in \mathcal{V}_{j}^{0} \\
\operatorname{Tr}_{j} u_{j}^{n+1}=\zeta^{n}
\end{array}\right.
$$

then

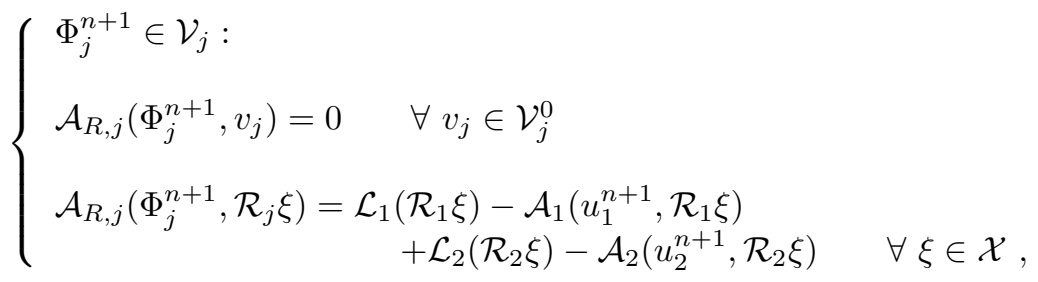


finally set

$$
\zeta^{n+1}=\zeta^{n}+\theta\left(\operatorname{Tr}_{1} \Phi_{1}^{n+1}+\operatorname{Tr}_{2} \Phi_{2}^{n+1}\right)
$$

We recall that $\mathcal{A}_{j}(\cdot, \cdot)=\mathcal{A}_{R, j}(\cdot, \cdot)+i \mathcal{A}_{I, j}(\cdot, \cdot)$, where $\mathcal{A}_{R, j}$ and $\mathcal{A}_{I, j}$ are Hermitian symmetric and continuous bilinear forms and $\mathcal{A}_{R, j}$ is coercive.

In order to write the iteration in terms of a preconditioned Richardson method for the Steklov-Poincaré problem (11), we consider a new operator associated to the bilinear forms $\mathcal{A}_{R, j}(\cdot, \cdot)$. For $j=1,2$, let us define the extension $\mathcal{F}_{j}: \mathcal{X} \rightarrow \mathcal{V}_{j}$ such that for all $\xi \in \mathcal{X}, \mathcal{F}_{j} \xi$ is the unique solution of

$$
\left\{\begin{array}{l}
\mathcal{F}_{j} \xi \in \mathcal{V}_{j}: \\
\mathcal{A}_{R, j}\left(\mathcal{F}_{j} \xi, v_{j}\right)=0 \quad \forall v_{j} \in \mathcal{V}_{j}^{0} \\
\operatorname{Tr}_{j}\left(\mathcal{F}_{j} \xi\right)=\xi
\end{array}\right.
$$

Then $\widehat{\mathcal{S}}_{R, j}: \mathcal{X} \rightarrow \mathcal{X}^{\prime}$ is defined as

$$
\left\langle\widehat{\mathcal{S}}_{R, j} \xi, \nu\right\rangle:=\mathcal{A}_{R, j}\left(\mathcal{F}_{j} \xi, \mathcal{F}_{j} \nu\right) \quad \forall \xi, \nu \in \mathcal{X}
$$

It is easy to see that

therefore

$$
\widehat{\mathcal{S}}_{R, j}\left(\operatorname{Tr}_{j} \Phi_{j}^{n+1}\right)=\Upsilon-\left(\mathcal{S}_{1}+\mathcal{S}_{2}\right) \zeta^{n}
$$

$$
\zeta^{n+1}=\zeta^{n}+\theta\left(\widehat{\mathcal{S}}_{R, 1}^{-1}+\widehat{\mathcal{S}}_{R, 2}^{-1}\right)\left[\Upsilon-\mathcal{S} \zeta^{n}\right]
$$

Then the iterative procedure $(18-20)$ is equivalent to the Richardson method for problem $(11)$ using $\left(\widehat{\mathcal{S}}_{R, 1}^{-1}+\right.$ $\left.\widehat{\mathcal{S}}_{R, 2}^{-1}\right)^{-1}$ as preconditioner. The iterative operator in (20) is given by

$$
\widehat{\mathcal{T}}_{\theta}=I-\theta\left(\widehat{\mathcal{S}}_{R, 1}^{-1}+\widehat{\mathcal{S}}_{R, 2}^{-1}\right) \mathcal{S}
$$

\section{Convergence Results}

\subsection{The $\gamma$-Dirichlet/Robin method}

In order to prove the convergence of the $\gamma$-Dirichlet/Robin method we prove the following abstract result.

Theorem 4.1. Let $\mathcal{X}$ be a complex Hilbert space, $\mathcal{X}^{\prime}$ its dual space and $\mathcal{Q}, \mathcal{Q}_{2}: \mathcal{X} \rightarrow \mathcal{X}^{\prime}$ linear operators. We assume that

1. $\mathcal{Q}, \mathcal{Q}_{2}$ are continuous, i.e.,

1.a: there exist $\beta>0$ such that

$$
|\langle\mathcal{Q} \eta, \lambda\rangle| \leq \beta\|\eta\|_{\mathcal{X}}\|\lambda\|_{\mathcal{X}} \quad \forall \eta, \lambda \in \mathcal{X}
$$

1.b: there exist $\beta_{2}>0$ such that

$$
\left|\left\langle\mathcal{Q}_{2} \eta, \lambda\right\rangle\right| \leq \beta_{2}\|\eta\|_{\mathcal{X}}\|\lambda\|_{\mathcal{X}} \quad \forall \eta, \lambda \in \mathcal{X}
$$

2. $\mathcal{Q}_{2}$ is real coercive, i.e., there exists $\alpha_{R 2}>0$ such that

$$
\operatorname{Re}\left\langle\mathcal{Q}_{2} \eta, \eta\right\rangle \geq \alpha_{R 2}\|\eta\|_{\mathcal{X}}^{2} \quad \forall \eta \in \mathcal{X} ;
$$


3. there exists a constant $\kappa^{*}>0$ such that

$$
\operatorname{Re}\left[\left\langle\mathcal{Q}_{2} \eta, \mathcal{Q}_{2}^{-1} \mathcal{Q} \eta\right\rangle+\langle\mathcal{Q} \eta, \eta\rangle\right] \geq \kappa^{*}\|\eta\|_{\mathcal{X}}^{2} \quad \forall \eta \in \mathcal{X} .
$$

Then for any $\eta^{0}$ in $\mathcal{X}$ the sequence

converges to 0 in $\mathcal{X}$, provided that

$$
\eta^{n+1}=\eta^{n}-\theta \mathcal{Q}_{2}^{-1} \mathcal{Q} \eta^{n}
$$

$$
0<\theta<\frac{\kappa^{*} \alpha_{R 2}^{2}}{\beta_{2} \beta^{2}}
$$

Proof. First we notice that $\mathcal{Q}_{2}$ is coercive because

$$
\left|\left\langle\mathcal{Q}_{2} \eta, \eta\right\rangle\right| \geq\left|\operatorname{Re}\left\langle\mathcal{Q}_{2} \eta, \eta\right\rangle\right| \geq \alpha_{R 2}\|\eta\|_{\mathcal{X}}^{2} .
$$

Hence from 1.b and 2 there exists $\mathcal{Q}_{2}^{-1}$.

We introduce the scalar product

$$
(\eta, \lambda)_{\mathcal{Q}_{2}}:=\frac{1}{2}\left(\left\langle\mathcal{Q}_{2} \eta, \lambda\right\rangle+\overline{\left\langle\mathcal{Q}_{2} \lambda, \eta\right\rangle}\right)
$$

with the corresponding norm

$$
\|\eta\|_{\mathcal{Q}_{2}}^{2}:=(\eta, \eta)_{\mathcal{Q}_{2}}=\operatorname{Re}\left\langle\mathcal{Q}_{2} \eta, \eta\right\rangle,
$$

which is equivalent to the norm $\|\eta\|_{\mathcal{X}}$, i.e.;

$$
\alpha_{R 2}\|\eta\|_{\mathcal{X}}^{2} \leq\|\eta\|_{\mathcal{Q}_{2}}^{2} \leq \beta_{2}\|\eta\|_{\mathcal{X}}^{2} .
$$

We shall prove that, choosing $\theta$ in a suitable interval, the map $T_{\theta}: \mathcal{X} \rightarrow \mathcal{X}$ defined as

$$
T_{\theta} \eta:=\eta-\theta \mathcal{Q}_{2}^{-1} \mathcal{Q} \eta
$$

is a contraction with respect to the norm $\|\cdot\|_{\mathcal{Q}_{2}}$. Assuming that $0 \leq \theta$, we have

$$
\begin{aligned}
\left\|T_{\theta} \eta\right\|_{\mathcal{Q}_{2}}^{2} & =\operatorname{Re}\left[\left\langle\mathcal{Q}_{2} \eta, \eta\right\rangle+\theta^{2}\left\langle\mathcal{Q} \eta, \mathcal{Q}_{2}^{-1} \mathcal{Q} \eta\right\rangle-\theta\left(\left\langle\mathcal{Q}_{2} \eta, \mathcal{Q}_{2}^{-1} \mathcal{Q} \eta\right\rangle+\langle\mathcal{Q} \eta, \eta\rangle\right)\right] \\
& \leq\|\eta\|_{\mathcal{Q}_{2}}^{2}+\theta^{2} \frac{\beta^{2}}{\alpha_{R 2}}\|\eta\|_{\mathcal{X}}^{2}-\theta \kappa^{*}\|\eta\|_{\mathcal{X}}^{2} \\
& \leq\left(1+\theta^{2} \frac{\beta^{2}}{\alpha_{R 2}^{2}}-\theta \frac{\kappa^{*}}{\beta_{2}}\right)\|\eta\|_{\mathcal{Q}_{2}}^{2} .
\end{aligned}
$$

By imposing the condition

the thesis follows.

$$
1+\theta^{2} \frac{\beta^{2}}{\alpha_{R 2}^{2}}-\theta \frac{\kappa^{*}}{\beta_{2}}<1
$$

Now, we are in position to prove

Theorem 4.2. Assuming that for $j=1,2$

H1. $\mathcal{A}_{j}: \mathcal{V}_{j} \times \mathcal{V}_{j} \rightarrow \mathbb{R}$ is a bilinear form such that

$$
\mathcal{A}_{j}(\cdot, \cdot)=\mathcal{A}_{R, j}(\cdot, \cdot)+i \mathcal{A}_{I, j}(\cdot, \cdot),
$$


where $\mathcal{A}_{R, j}$ and $\mathcal{A}_{I, j}$ are Hermitian symmetric and continuous bilinear forms, i.e., there exist constants $\Lambda_{L j}>0$ such that

$$
\mathcal{A}_{L, j}\left(v_{j}, w_{j}\right) \leq \Lambda_{L j}\left\|v_{j}\right\| \mathcal{V}_{j}\left\|w_{j}\right\|_{\mathcal{V}_{j}} \quad \forall v_{j}, w_{j} \in \mathcal{V}_{j}, L=R, I, j=1,2
$$

and $\mathcal{A}_{R, j}$ is coercive, i.e., there exist constants $\Delta_{R j}>0$ such that

$$
\mathcal{A}_{R, j}\left(v_{j}, v_{j}\right) \geq \Delta_{R j}\left\|v_{j}\right\|_{\mathcal{V}_{j}}^{2} \quad \forall v_{j} \in \mathcal{V}_{j}, j=1,2
$$

H2. the trace operator $\operatorname{Tr}_{j}: \mathcal{V}_{j} \rightarrow \mathcal{X}$ is continuous, i.e., there exist constants $C_{T_{j}}>0$ such that

$$
\left\|\operatorname{Tr}_{j} v_{j}\right\|_{\mathcal{X}} \leq C_{T r_{j}}\left\|v_{j}\right\|_{\mathcal{V}_{j}} \quad \forall v_{j} \in \mathcal{V}_{j}, j=1,2
$$

H3. there exist extension operators $R_{j}: \mathcal{X} \rightarrow \mathcal{V}_{j}$, which are continuous, i.e., there exist constants $C_{R_{j}}>0$ such that

$$
\left\|R_{j} \eta\right\|_{\mathcal{V}_{j}} \leq C_{R_{j}}\|\eta\|_{\mathcal{X}} \quad \forall \eta \in \mathcal{X}, j=1,2,
$$

then there exists $\gamma^{*} \in \mathbb{R}$ such that for each $\gamma \geq 0, \gamma>\gamma^{*}$ and for each $\zeta^{0} \in \mathcal{X}$ the iterative scheme (12-14) is convergent in $\mathcal{X}$, provided that the relaxation parameter $\theta$ is chosen in a suitable interval $\left(0, \theta_{\gamma}\right)$.

Proof. We apply Theorem 4.1 taking

$$
\begin{array}{ll}
\langle\mathcal{Q} \eta, \lambda\rangle=\mathcal{A}_{1}\left(\mathcal{E}_{1} \eta, \mathcal{E}_{1} \lambda\right)+\mathcal{A}_{2}\left(\mathcal{E}_{2} \eta, \mathcal{E}_{2} \lambda\right) & \left(\mathcal{Q}=\mathcal{S}_{1}+\mathcal{S}_{2}\right) \\
\left\langle\mathcal{Q}_{2} \eta, \lambda\right\rangle=\mathcal{A}_{2}\left(\mathcal{E}_{2} \eta, \mathcal{E}_{2} \lambda\right)+\gamma(\eta, \lambda)_{\mathcal{X}} & \left(\mathcal{Q}_{2}=\mathcal{S}_{2}^{\gamma}\right)
\end{array}
$$

In particular,

$$
\operatorname{Re}\left\langle\mathcal{Q}_{2} \eta, \eta\right\rangle=\mathcal{A}_{R, 2}\left(\mathcal{E}_{2} \eta, \mathcal{E}_{2} \eta\right)+\gamma(\eta, \eta)_{\mathcal{X}}
$$

We notice that from H1 and H3 the extension operator used in the definition of the Steklov-Poincaré operator is continuous; in fact,

$$
\Delta_{R j}\left\|\mathcal{E}_{j} \eta\right\|_{\mathcal{V}_{j}}^{2} \leq\left|\mathcal{A}_{j}\left(\mathcal{E}_{j} \eta, \mathcal{E}_{j} \eta\right)\right|=\left|\mathcal{A}_{j}\left(\mathcal{E}_{j} \eta, R_{j} \eta\right)\right| \leq\left(\Lambda_{R j}+\Lambda_{I j}\right)\left\|\mathcal{E}_{j} \eta\right\| \mathcal{V}_{j}\left\|R_{j} \eta\right\| \mathcal{V}_{j}
$$

SO

$$
\left\|\mathcal{E}_{j} \eta\right\|_{\mathcal{V}_{j}} \leq C_{\mathcal{E}_{j}}\|\eta\|_{\mathcal{X}}
$$

with $C_{\mathcal{E}_{j}}=\frac{\Lambda_{R j}+\Lambda_{I j}}{\Delta_{R j}} C_{R_{j}}$. Hence it is clear that assumptions 1 and 2 of Theorem 4.1 are satisfied with constants

$$
\begin{aligned}
& \beta=\left(\Lambda_{R 1}+\Lambda_{I 1}\right) C_{\mathcal{E}_{1}}^{2}+\left(\Lambda_{R 2}+\Lambda_{I 2}\right) C_{\mathcal{E}_{2}}^{2} \\
& \beta_{2}=\left(\Lambda_{R 2}+\Lambda_{I 2}\right) C_{\mathcal{E}_{2}}^{2}+\gamma=: \tilde{\beta}_{2}+\gamma \\
& \alpha_{R 2}=\frac{\Delta_{R 2}}{C_{T r_{2}}^{2}}+\gamma=: \tilde{\alpha}_{R 2}+\gamma .
\end{aligned}
$$

We notice that $\beta, \tilde{\beta}_{2}$ and $\tilde{\alpha}_{R 2}$ are independent of $\gamma$. 
Now we shall prove that there exists $\gamma^{*} \in \mathbb{R}$ such that for each $\gamma>\gamma^{*}$, and $\gamma \geq 0$ assumption 3 of Theorem 4.1 is satisfied. We notice that

$$
\begin{aligned}
\operatorname{Re}\left[\left\langle\mathcal{Q}_{2} \eta\right.\right. & \left.\left., \mathcal{Q}_{2}^{-1} \mathcal{Q} \eta\right\rangle+\langle\mathcal{Q} \eta, \eta\rangle\right] \\
& =\operatorname{Re}\left[\left\langle\mathcal{Q}_{2} \eta, \mathcal{Q}_{2}^{-1} \mathcal{Q} \eta\right\rangle-\overline{\langle\mathcal{Q} \eta, \eta\rangle}\right]+\operatorname{Re}[\overline{\langle\mathcal{Q} \eta, \eta\rangle}+\langle\mathcal{Q} \eta, \eta\rangle] \\
& =2 \operatorname{Re}\langle\mathcal{Q} \eta, \eta\rangle+\operatorname{Re}\left[\left\langle\mathcal{Q}_{2} \eta, \mathcal{Q}_{2}^{-1} \mathcal{Q} \eta\right\rangle-\overline{\langle\mathcal{Q} \eta, \eta\rangle}\right] \\
& \geq 2 \operatorname{Re}\langle\mathcal{Q} \eta, \eta\rangle-\left|\operatorname{Re}\left[\left\langle\mathcal{Q}_{2} \eta, \mathcal{Q}_{2}^{-1} \mathcal{Q} \eta\right\rangle-\overline{\langle\mathcal{Q} \eta, \eta\rangle}\right]\right| .
\end{aligned}
$$

Moreover

$$
\operatorname{Re}\langle\mathcal{Q} \eta, \eta\rangle=\mathcal{A}_{R 1}\left(\mathcal{E}_{1} \eta, \mathcal{E}_{1} \eta\right)+\mathcal{A}_{R 2}\left(\mathcal{E}_{2} \eta, \mathcal{E}_{2} \eta\right) \geq\left(\frac{\Delta_{R 1}}{C_{T r_{1}}^{2}}+\frac{\Delta_{R 2}}{C_{T r_{2}}^{2}}\right)\|\eta\|_{\mathcal{X}}^{2}=\alpha\|\eta\|_{\mathcal{X}}^{2},
$$

where we have set $\alpha:=\frac{\Delta_{R 1}}{C_{T r_{1}}^{2}}+\frac{\Delta_{R 2}}{C_{T r_{2}}^{2}}$. Taking $\lambda=\mathcal{Q}_{2}^{-1} \mathcal{Q} \eta$ we have, by a straightforward computation,

$$
\begin{aligned}
\mid \operatorname{Re} & {\left[\left\langle\mathcal{Q}_{2} \eta, \mathcal{Q}_{2}^{-1} \mathcal{Q} \eta\right\rangle-\overline{\langle\mathcal{Q} \eta, \eta\rangle}\right]|=| \operatorname{Re}\left[\left\langle\mathcal{Q}_{2} \eta, \lambda\right\rangle-\overline{\left\langle\mathcal{Q}_{2} \lambda, \eta\right\rangle}\right] \mid } \\
& =\left|\operatorname{Re}\left[\mathcal{A}_{2}\left(\mathcal{E}_{2} \eta, \mathcal{E}_{2} \lambda\right)+\gamma(\eta, \lambda)_{\mathcal{X}}-\overline{\mathcal{A}_{2}\left(\mathcal{E}_{2} \lambda, \mathcal{E}_{2} \eta\right)}-\gamma \overline{(\lambda, \eta)_{\mathcal{X}}}\right]\right| \\
& =\left|\operatorname{Re}\left[2 i \mathcal{A}_{I, 2}\left(\mathcal{E}_{2} \eta, \mathcal{E}_{2} \lambda\right)\right]\right| \leq 2\left|\mathcal{A}_{I, 2}\left(\mathcal{E}_{2} \eta, \mathcal{E}_{2} \lambda\right)\right| \\
& \leq 2 \Lambda_{I 2} C_{\mathcal{E}_{2}}^{2}\|\eta\|_{\mathcal{X}}\|\lambda\|_{\mathcal{X}}=2 \beta_{I 2}\|\eta\|_{\mathcal{X}}\|\lambda\|_{\mathcal{X}},
\end{aligned}
$$

where we have set $\beta_{I 2}:=\Lambda_{I 2} C_{\mathcal{E}_{2}}^{2}$. Since $\|\lambda\|_{\mathcal{X}}=\left\|\mathcal{Q}_{2}^{-1} \mathcal{Q} \eta\right\|_{\mathcal{X}} \leq \frac{\beta}{\alpha_{R 2}}\|\eta\|_{\mathcal{X}}$, we have

$$
\operatorname{Re}\left[\left\langle\mathcal{Q}_{2} \eta, \mathcal{Q}_{2}^{-1} \mathcal{Q} \eta\right\rangle+\langle\mathcal{Q} \eta, \eta\rangle\right] \geq 2\left(\alpha-\beta_{I 2} \frac{\beta}{\alpha_{R 2}}\right)\|\eta\|_{\mathcal{X}}^{2}
$$

We notice that $\alpha, \beta$ and $\beta_{I 2}$ do not depend on $\gamma$ and that $\alpha_{R 2}=\tilde{\alpha}_{R 2}+\gamma$ with $\tilde{\alpha}_{R 2}>0$ independent of $\gamma$. Hence, taking $\gamma \geq 0$ and

$$
\gamma>\frac{\beta_{I 2} \beta}{\alpha}-\tilde{\alpha}_{R 2}=: \gamma^{*}
$$

assumption 3 of Theorem 4.1 is satisfied with

$$
\kappa^{*}(\gamma)=2\left(\alpha-\beta_{I 2} \frac{\beta}{\tilde{\alpha}_{R 2}+\gamma}\right)>0 .
$$

Provided that $\theta \in\left(0, \theta_{\gamma}\right)$ with

$$
\theta_{\gamma}=2 \frac{\alpha\left(\tilde{\alpha}_{R 2}+\gamma\right)-\beta_{I 2} \beta}{\left(\tilde{\beta}_{2}+\gamma\right) \beta^{2}}\left(\tilde{\alpha}_{R 2}+\gamma\right),
$$

the iterative scheme $(12-14)$ is convergent in $\mathcal{X}$.

\subsection{The $\gamma$-Robin/Robin method}

We state an abstract result that will be used to prove the convergence of the $\gamma$-Robin/Robin method.

Theorem 4.3. Let $\mathcal{X}$ be a complex Hilbert space, $\mathcal{X}^{\prime}$ its dual space and $\mathcal{Q}, \mathcal{Q}_{1}, \mathcal{Q}_{2}: \mathcal{X} \rightarrow \mathcal{X}^{\prime}$ linear operators. We assume that

1. $\mathcal{Q}$ and $\mathcal{Q}_{j}$ for $j=1,2$, are continuous, i.e., 
1.a: there exist $\beta>0$ such that

$$
|\langle\mathcal{Q} \eta, \lambda\rangle| \leq \beta\|\eta\|_{\mathcal{X}}\|\lambda\|_{\mathcal{X}} \quad \forall \eta, \lambda \in \mathcal{X}
$$

1.b: there exist $\beta_{j}>0$ such that

$$
\left|\left\langle\mathcal{Q}_{j} \eta, \lambda\right\rangle\right| \leq \beta_{j}\|\eta\|_{\mathcal{X}}\|\lambda\|_{\mathcal{X}} \quad \forall \eta, \lambda \in \mathcal{X}
$$

2. $\mathcal{Q}_{j}$ is real coercive for $j=1,2$, i.e., there exist $\alpha_{R j}>0$ such that

$$
\operatorname{Re}\left\langle\mathcal{Q}_{j} \eta, \eta\right\rangle \geq \alpha_{R j}\|\eta\|_{\mathcal{X}}^{2} \quad \forall \eta \in \mathcal{X}
$$

Assume moreover that the operator $\mathcal{N}:=\left(\mathcal{Q}_{1}^{-1}+\mathcal{Q}_{2}^{-1}\right)^{-1}$ satisfies the condition

3. there exists a constant $\kappa^{*}>0$ such that

$$
\operatorname{Re}\left[\left\langle\mathcal{N} \eta, \mathcal{N}^{-1} \mathcal{Q} \eta\right\rangle+\langle\mathcal{Q} \eta, \eta\rangle\right] \geq \kappa^{*}\|\eta\|_{\mathcal{X}}^{2} \quad \forall \eta \in \mathcal{X}
$$

Then there exists $\theta^{0}>0$ such that for each $\theta \in\left(0, \theta^{0}\right)$ and for any given $\eta^{0}$ in $\mathcal{X}$ the sequence

$$
\eta^{n+1}=\eta^{n}-\theta \mathcal{N}^{-1} \mathcal{Q} \eta^{n}
$$

converges to 0 in $\mathcal{X}$.

Proof. As in Theorem 4.1 we notice that, since $\mathcal{Q}_{j}$ is real coercive, it is coercive. Hence $\mathcal{N}$ is well defined and it is continuous and coercive (see, e.g. [23], p. 108); more precisely,

$$
|\langle\mathcal{N} \eta, \lambda\rangle| \leq \beta_{\mathcal{N}}\|\eta\|_{\mathcal{X}}\|\lambda\|_{\mathcal{X}} \quad \forall \eta, \lambda \in \mathcal{X}
$$

with $\beta_{\mathcal{N}}=\left(\frac{\alpha_{R 1}}{\beta_{1}^{2}}+\frac{\alpha_{R 2}}{\beta_{2}^{2}}\right)^{-1}$, and

$$
|\langle\mathcal{N} \eta, \eta\rangle| \geq \alpha_{\mathcal{N}}\|\eta\|_{\mathcal{X}}^{2} \quad \forall \eta \in \mathcal{X}
$$

with $\alpha_{\mathcal{N}}=\left(\frac{\alpha_{R 1}}{\beta_{1}^{2}}+\frac{\alpha_{R 2}}{\beta_{2}^{2}}\right)\left(\frac{1}{\alpha_{R 1}}+\frac{1}{\alpha_{R 2}}\right)^{-2}$.

Now we shall prove that the following bilinear form

$$
(\eta, \lambda)_{\mathcal{N}}:=\frac{1}{2}(\langle\mathcal{N} \eta, \lambda\rangle+\overline{\langle\mathcal{N} \lambda, \eta\rangle}) \quad \forall \eta, \lambda \in \mathcal{X}
$$

is a scalar product in $\mathcal{X}$ and that the corresponding norm

$$
\|\eta\|_{\mathcal{N}}:=[\operatorname{Re}\langle\mathcal{N} \eta, \eta\rangle]^{1 / 2}
$$

is equivalent to the norm $\|\eta\|_{\mathcal{X}}$. 
Given $\eta \in \mathcal{X}$ we set $\psi=\mathcal{N} \eta$ and for $j=1,2, \lambda_{j}=\mathcal{Q}_{j}^{-1} \psi$. Then

$$
\begin{aligned}
\|\eta\|_{\mathcal{N}}^{2} & =\operatorname{Re}\langle\mathcal{N} \eta, \eta\rangle=\operatorname{Re}\left\langle\psi,\left(\mathcal{Q}_{1}^{-1}+\mathcal{Q}_{2}^{-1}\right) \psi\right\rangle \\
& =\operatorname{Re}\left[\left\langle\mathcal{Q}_{1} \lambda_{1}, \lambda_{1}\right\rangle+\left\langle\mathcal{Q}_{2} \lambda_{2}, \lambda_{2}\right\rangle\right] \\
& \geq \alpha_{R 1}\left\|\lambda_{1}\right\|_{\mathcal{X}}^{2}+\alpha_{R 2}\left\|\lambda_{2}\right\|_{\mathcal{X}}^{2}=\alpha_{R 1}\left\|\mathcal{Q}_{1}^{-1} \psi\right\|_{\mathcal{X}}^{2}+\alpha_{R 2}\left\|\mathcal{Q}_{2}^{-1} \psi\right\|_{\mathcal{X}}^{2} \\
& \geq\left(\frac{\alpha_{R 1}}{\beta_{1}^{2}}+\frac{\alpha_{R 2}}{\beta_{2}^{2}}\right)\|\psi\|_{\mathcal{X}^{\prime}}^{2}=\frac{1}{\beta_{\mathcal{N}}}\|\mathcal{N} \eta\|_{\mathcal{X}^{\prime}}^{2} \\
& \geq \frac{\alpha_{\mathcal{N}}^{2}}{\beta_{\mathcal{N}}}\|\eta\|_{\mathcal{X}}^{2} .
\end{aligned}
$$

Moreover

$$
\|\eta\|_{\mathcal{N}}^{2}=\operatorname{Re}\langle\mathcal{N} \eta, \eta\rangle \leq|\langle\mathcal{N} \eta, \eta\rangle| \leq \beta_{\mathcal{N}}\left\|_{\eta}\right\|_{\mathcal{X}}^{2}
$$

hence

$$
\frac{\alpha_{\mathcal{N}}^{2}}{\beta_{\mathcal{N}}}\|\eta\|_{\mathcal{X}}^{2} \leq\|\eta\|_{\mathcal{N}}^{2} \leq \beta_{\mathcal{N}}\|\eta\|_{\mathcal{X}}^{2}
$$

As in Theorem 4.1, in order to prove the convergence of $\eta^{n}$, we show that the map $\widetilde{T}_{\theta}: \mathcal{X} \rightarrow \mathcal{X}$ defined as

$$
\widetilde{T}_{\theta} \eta:=\eta-\theta \mathcal{N}^{-1} \mathcal{Q} \eta
$$

is a contraction with respect to the norm $\|\cdot\|_{\mathcal{N}}$. Assuming that $0 \leq \theta$, we have

$$
\begin{aligned}
\left\|\widetilde{T}_{\theta} \eta\right\|_{\mathcal{N}}^{2} & =\operatorname{Re}\left[\langle\mathcal{N} \eta, \eta\rangle+\theta^{2}\left\langle\mathcal{Q} \eta, \mathcal{N}^{-1} \mathcal{Q} \eta\right\rangle-\theta\left(\left\langle\mathcal{N} \eta, \mathcal{N}^{-1} \mathcal{Q} \eta\right\rangle+\langle\mathcal{Q} \eta, \eta\rangle\right)\right] \\
& \leq\|\eta\|_{\mathcal{N}}^{2}+\theta^{2} \frac{\beta^{2}}{\alpha_{\mathcal{N}}}\|\eta\|_{\mathcal{X}}^{2}-\theta \kappa^{*}\|\eta\|_{\mathcal{X}}^{2} \\
& \leq\left(1+\theta^{2} \frac{\beta^{2} \beta_{\mathcal{N}}}{\alpha_{\mathcal{N}}^{3}}-\theta \frac{\kappa^{*}}{\beta_{\mathcal{N}}}\right)\|\eta\|_{\mathcal{N}}^{2}
\end{aligned}
$$

Setting $\theta^{0}=\frac{\kappa^{*} \alpha_{\mathcal{N}}^{3}}{\beta^{2} \beta_{\mathcal{N}}^{2}}$ we conclude that $\widetilde{T}_{\theta}$ is a contraction for all $\theta \in\left(0, \theta^{0}\right)$.

Applying Theorem 4.3 we can prove the convergence of the $\gamma$-Robin/Robin method.

Theorem 4.4. Assuming that H1, H2 and H3 in Theorem 4.2 hold, there exists $\gamma^{\sharp} \in \mathbb{R}$ such that for each $\gamma \geq 0, \gamma>\gamma^{\sharp}$ and for each $\zeta^{0} \in \mathcal{X}$ the iterative scheme (15-17) is convergent in $\mathcal{X}$ provided that the relaxation parameter $\theta$ is chosen in a suitable interval $\left(0, \theta_{\gamma}^{0}\right)$.

Proof. We apply Theorem 4.3 taking

$$
\begin{array}{ll}
\langle\mathcal{Q} \eta, \lambda\rangle=\mathcal{A}_{1}\left(\mathcal{E}_{1} \eta, \mathcal{E}_{1} \lambda\right)+\mathcal{A}_{2}\left(\mathcal{E}_{2} \eta, \mathcal{E}_{2} \lambda\right) & \left(\mathcal{Q}=\mathcal{S}_{1}+\mathcal{S}_{2}\right) \\
\left\langle\mathcal{Q}_{j} \eta, \lambda\right\rangle=\mathcal{A}_{j}\left(\mathcal{E}_{j} \eta, \mathcal{E}_{j} \lambda\right)+\gamma(\eta, \lambda)_{\mathcal{X}} & \left(\mathcal{Q}_{j}=\mathcal{S}_{j}^{\gamma}\right)
\end{array}
$$


for $j=1,2$. As in Theorem 4.2 is easy to see that assumptions 1 and 2 of Theorem 4.3 are satisfied with constants

$$
\begin{aligned}
& \beta=\left(\Lambda_{R 1}+\Lambda_{I 1}\right) C_{\mathcal{E}_{1}}^{2}+\left(\Lambda_{R 2}+\Lambda_{I 2}\right) C_{\mathcal{E}_{2}}^{2}, \\
& \beta_{j}=\left(\Lambda_{R j}+\Lambda_{I j}\right) C_{\mathcal{E}_{j}}^{2}+\gamma=: \tilde{\beta}_{j}+\gamma, \\
& \alpha_{R j}=\frac{\Delta_{R j}}{C_{T r_{j}}^{2}}+\gamma=: \tilde{\alpha}_{R j}+\gamma,
\end{aligned}
$$

$j=1,2$. Note that $\beta, \tilde{\beta}_{j}$ and $\tilde{\alpha}_{R j}$ are independent of $\gamma$. Hence we shall show that there exists $\gamma^{\sharp} \in \mathbb{R}$ such that for $\gamma>\gamma^{\sharp}$ and $\gamma \leq 0$, assumption 3 of Theorem 4.3 is satisfied.

Setting $\lambda_{j}=\mathcal{Q}_{j}^{-1} \mathcal{N} \eta, \nu_{j}=\mathcal{Q}_{j}^{-1} \mathcal{Q} \eta$ we have

$$
\begin{aligned}
\left|\operatorname{Re} e\left[\left\langle\mathcal{N} \eta, \mathcal{N}^{-1} \mathcal{Q} \eta\right\rangle-\overline{\langle\mathcal{Q} \eta, \eta\rangle}\right]\right| \\
\quad=\left|\operatorname{Re}\left[\left\langle\mathcal{Q}_{1} \lambda_{1}, \nu_{1}\right\rangle+\left\langle\mathcal{Q}_{2} \lambda_{2}, \nu_{2}\right\rangle-\overline{\left\langle\mathcal{Q}_{1} \nu_{1}, \lambda_{1}\right\rangle}-\overline{\left\langle\mathcal{Q}_{2} \nu_{2}, \lambda_{2}\right\rangle}\right]\right| \\
\quad=\left|\operatorname{Re}\left[2 i \mathcal{A}_{I, 1}\left(\mathcal{E}_{1} \lambda_{1}, \mathcal{E}_{1} \nu_{1}\right)+2 i \mathcal{A}_{I, 2}\left(\mathcal{E}_{2} \lambda_{2}, \mathcal{E}_{2} \nu_{2}\right)\right]\right| \\
\quad \leq 2\left|\mathcal{A}_{I, 1}\left(\mathcal{E}_{1} \lambda_{1}, \mathcal{E}_{1} \nu_{1}\right)+\mathcal{A}_{I, 2}\left(\mathcal{E}_{2} \lambda_{2}, \mathcal{E}_{2} \nu_{2}\right)\right| \\
\quad \leq 2\left(\Lambda_{I 1} C_{\mathcal{E}_{1}}^{2}\left\|\mathcal{Q}_{1}^{-1} \mathcal{N} \eta\right\|_{\mathcal{X}}\left\|\mathcal{Q}_{1}^{-1} \mathcal{Q} \eta\right\|_{\mathcal{X}}+\Lambda_{I 2} C_{\mathcal{E}_{2}}^{2}\left\|\mathcal{Q}_{2}^{-1} \mathcal{N} \eta\right\|_{\mathcal{X}}\left\|\mathcal{Q}_{2}^{-1} \mathcal{Q} \eta\right\|_{\mathcal{X}}\right) \\
\quad \leq 2\left(\frac{\Lambda_{I 1} C_{\mathcal{E}_{1}}^{2}}{\alpha_{R 1}^{2}}+\frac{\Lambda_{I 2} C_{\mathcal{E}_{2}}^{2}}{\alpha_{R 2}^{2}}\right)\|\mathcal{N} \eta\|_{\mathcal{X}}\|\mathcal{Q} \eta\|_{\mathcal{X}} \\
\quad \leq 2\left(\frac{\Lambda_{I 1} C_{\mathcal{E}_{1}}^{2}}{\alpha_{R 1}^{2}}+\frac{\Lambda_{I 2} C_{\mathcal{E}_{2}}^{2}}{\alpha_{R 2}^{2}}\right) \beta_{\mathcal{N}} \beta\|\eta\|_{\mathcal{X}}^{2} .
\end{aligned}
$$

Hence, setting $\beta_{I j}:=\Lambda_{I j} C_{\mathcal{E}_{j}}^{2}$

$$
\left|R e\left[\left\langle\mathcal{N} \eta, \mathcal{N}^{-1} \mathcal{Q} \eta\right\rangle+\langle\mathcal{Q} \eta, \eta\rangle\right]\right| \geq 2\left[\alpha-\left(\frac{\beta_{I 1}}{\alpha_{R 1}^{2}}+\frac{\beta_{I 2}}{\alpha_{R 2}^{2}}\right) \beta_{\mathcal{N}} \beta\right]\|\eta\|_{\mathcal{X}}^{2}=2 \kappa^{*}(\gamma)\|\eta\|_{\mathcal{X}}^{2}
$$

where

$$
\kappa^{*}(\gamma)=2\left[\alpha-\left(\frac{\beta_{I 1}}{\left(\tilde{\alpha}_{R 1}+\gamma\right)^{2}}+\frac{\beta_{I 2}}{\left(\tilde{\alpha}_{R 2}+\gamma\right)^{2}}\right)\left(\frac{\tilde{\alpha}_{R 1}+\gamma}{\left(\tilde{\beta}_{1}+\gamma\right)^{2}}+\frac{\tilde{\alpha}_{R 2}+\gamma}{\left(\tilde{\beta}_{2}+\gamma\right)^{2}}\right)^{-1} \beta\right],
$$

with $\alpha, \beta, \beta_{I j}, \tilde{\beta}_{j}$ and $\tilde{\alpha}_{R j}$ independent on $\gamma$. Since

$$
\left(\frac{\beta_{I 1}}{\left(\tilde{\alpha}_{R 1}+\gamma\right)^{2}}+\frac{\beta_{I 2}}{\left(\tilde{\alpha}_{R 2}+\gamma\right)^{2}}\right)\left(\frac{\tilde{\alpha}_{R 1}+\gamma}{\left(\tilde{\beta}_{1}+\gamma\right)^{2}}+\frac{\tilde{\alpha}_{R 2}+\gamma}{\left(\tilde{\beta}_{2}+\gamma\right)^{2}}\right)^{-1}
$$

tends to zero when $\gamma$ tends to infinity, there exist $\gamma^{\sharp}$ such that $\kappa^{*}(\gamma)>0$ for each $\gamma>\gamma^{\sharp}$.

\subsection{The modified Neumann/Neumann method}

We can also prove the following:

Theorem 4.5. Let $\mathcal{X}$ be a complex Hilbert space, $\mathcal{X}^{\prime}$ its dual space and $\mathcal{Q}, \mathcal{P}_{1}, \mathcal{P}_{2}: \mathcal{X} \rightarrow \mathcal{X}^{\prime}$ linear operators. Suppose that for $j=1,2, \mathcal{P}_{j}$ is Hermitian symmetric, i.e., $\left\langle\mathcal{P}_{j} \eta, \lambda\right\rangle=\overline{\left\langle\mathcal{P}_{j} \lambda, \eta\right\rangle}$ for each $\eta, \lambda \in \mathcal{X}$. We assume that 
1. $\mathcal{Q}$ is continuous, i.e., there exist $\beta>0$ such that

$$
|\langle\mathcal{Q} \eta, \lambda\rangle| \leq \beta\|\eta\|_{\mathcal{X}}\|\lambda\|_{\mathcal{X}} \quad \forall \eta, \lambda \in \mathcal{X} ;
$$

2. for $j=1,2, \mathcal{P}_{j}$ is continuous and coercive, i.e., 2.a: there exist $\beta_{P j}>0$ such that

$$
\left|\left\langle\mathcal{P}_{j} \eta, \lambda\right\rangle\right| \leq \beta_{P j}\|\eta\|_{\mathcal{X}}\|\lambda\|_{\mathcal{X}} \quad \forall \eta, \lambda \in \mathcal{X},
$$

2.b: there exist $\alpha_{P j}>0$ such that

$$
\left\langle\mathcal{P}_{j} \eta, \eta\right\rangle \geq \alpha_{P j}\|\eta\|_{\mathcal{X}}^{2} \quad \forall \eta \in \mathcal{X} ;
$$

3. $\mathcal{Q}$ is real coercive, i.e. there exist $\alpha_{R}>0$ such that

$$
\operatorname{Re}\langle\mathcal{Q} \eta, \eta\rangle \geq \alpha_{R}\|\eta\|_{\mathcal{X}}^{2} \quad \forall \eta \in \mathcal{X} .
$$

Then there exists $\theta_{0}$ such that for each $\theta \in\left(0, \theta_{0}\right)$ and for any $\eta^{0}$ in $\mathcal{X}$ the sequence

$$
\eta^{n+1}=\eta^{n}-\theta\left(\mathcal{P}_{1}^{-1}+\mathcal{P}_{2}^{-1}\right)^{-1} \mathcal{Q} \eta^{n}
$$

converges to 0 in $\mathcal{X}$.

The proof is similar to that of Theorem 4.3, taking $\mathcal{P}_{j}$ instead of $\mathcal{Q}_{j}$, and taking into account that the operators $\mathcal{P}_{j}$ are Hermitian symmetric.

Assuming that H1, $\mathrm{H} 2$ and $\mathrm{H} 3$ of Theorem 4.2 are satisfied, the convergence of the iterative scheme (18-20) can be proven by applying Theorem 4.5 with

$$
\begin{aligned}
& \mathcal{Q}=\mathcal{S}_{1}+\mathcal{S}_{2} \\
& \mathcal{P}_{j}=\widehat{\mathcal{S}}_{R, j}, \quad j=1,2 .
\end{aligned}
$$

\section{Application to time-harmonic Maxwell equations and other problems}

Going back to the discrete time-harmonic Maxwell problem, the $\gamma$-Dirichlet/Robin procedure reads: being given $\boldsymbol{\zeta}_{h}^{0} \in X_{\Gamma, h}$, for $n \geq 0$ solve

$$
\left\{\begin{array}{l}
\mathbf{E}_{1, h}^{n+1} \in V_{1, h}: \\
b_{1}\left(\mathbf{E}_{1, h}^{n+1}, \mathbf{v}_{1, h}\right)=L_{1}\left(\mathbf{v}_{1, h}\right) \quad \forall \mathbf{v}_{1, h} \in V_{1, h}^{0} \\
\operatorname{Tr}_{1} \mathbf{E}_{1, h}^{n+1}=\boldsymbol{\zeta}_{h}^{n},
\end{array}\right.
$$

then

$$
\left\{\begin{array}{l}
\mathbf{E}_{2, h}^{n+1} \in V_{2, h}: \\
b_{2}\left(\mathbf{E}_{2, h}^{n+1}, \mathbf{v}_{2, h}\right)=L_{2}\left(\mathbf{v}_{2, h}\right) \quad \forall \mathbf{v}_{2, h} \in V_{2, h}^{0} \\
b_{2}\left(\mathbf{E}_{2, h}^{n+1}, \mathbf{R}_{2, h} \boldsymbol{\xi}_{h}\right)+\gamma\left(\operatorname{Tr}_{2} \mathbf{E}_{2, h}^{n+1}, \boldsymbol{\xi}_{h}\right)_{X_{\Gamma}}=L_{2}\left(\mathbf{R}_{2, h} \boldsymbol{\xi}_{h}\right) \\
\quad+L_{1}\left(\mathbf{R}_{1, h} \boldsymbol{\xi}_{h}\right)-b_{1}\left(\mathbf{E}_{1, h}^{n+1}, \mathbf{R}_{1, h} \boldsymbol{\xi}_{h}\right)+\gamma\left(\operatorname{Tr}_{1} \mathbf{E}_{1, h}^{n+1}, \boldsymbol{\xi}_{h}\right)_{X_{\Gamma}} \quad \forall \boldsymbol{\xi}_{h} \in X_{\Gamma, h},
\end{array}\right.
$$


finally set

$$
\boldsymbol{\zeta}_{h}^{n+1}=(1-\theta) \boldsymbol{\zeta}_{h}^{n}+\theta \operatorname{Tr}_{2} \mathbf{E}_{2, h}^{n+1}
$$

Theorem 5.1. There exists a constant $\gamma^{*} \in \mathbb{R}$ and for each $\gamma \geq 0, \gamma>\gamma^{*}$ there exists an interval $I_{\gamma}=\left(0, \theta_{\gamma}\right)$ such that for each $\zeta_{h}^{0} \in X_{\Gamma, h}$ and for each $\theta \in I_{\gamma}$ the iterative procedure (22-24) converges. Moreover the rate of convergence is independent of $h$.

Proof. We are going to apply Theorem 4.2 with $\mathcal{V}_{j}=V_{j, h}, \mathcal{A}_{j}(\cdot, \cdot)=b_{j}(\cdot, \cdot)$ and $\mathcal{X}=X_{\Gamma, h}$. The assumptions $\mathrm{H} 1$ and $\mathrm{H} 2$ are trivially satisfied, while we need to verify that H3 holds, namely, to construct a suitable extension operator from $X_{\Gamma, h}$ to $V_{j, h}$ uniformly continuous with respect to $h$. In [3] such an extension operator is constructed when Nédélec finite elements of the first kind are used. (Indeed, the result in [3] concerns the interface condition $\mathbf{v} \times \mathbf{n}$ on $\Gamma$; the construction of an extension operator for the condition $\mathbf{n} \times(\mathbf{v} \times \mathbf{n})$ on $\Gamma$ can be done in a similar way.) An analogous construction is also possible using Nédélec finite elements of the second kind. The thesis follows from Theorem 4.2 .

If $Z=1-\mathrm{i} B$, multiplying the iteration $(22-24)$ by $Z^{-1}$ the iterative procedure can also be written in the following way: being given $\boldsymbol{\zeta}_{h}^{0} \in X_{\Gamma, h}$, for each $n \geq 0$ solve

$$
\left\{\begin{array}{l}
\mathbf{E}_{1, h}^{n+1} \in V_{1, h}: \\
a_{1}\left(\mathbf{E}_{1, h}^{n+1}, \mathbf{v}_{1, h}\right)=\int_{\Omega_{1}} \mathbf{F} \cdot \overline{\mathbf{v}}_{1, h} \quad \forall \mathbf{v}_{1, h} \in V_{1, h}^{0} \\
\operatorname{Tr}_{1} \mathbf{E}_{1, h}^{n+1}=\boldsymbol{\zeta}_{h}^{n}
\end{array}\right.
$$

then

$$
\left\{\begin{array}{l}
\mathbf{E}_{2, h}^{n+1} \in V_{2, h}: \\
a_{2}\left(\mathbf{E}_{2, h}^{n+1}, \mathbf{v}_{2, h}\right)=\int_{\Omega_{2}} \mathbf{F} \cdot \overline{\mathbf{v}}_{2, h} \quad \forall \mathbf{v}_{2, h} \in V_{2, h}^{0} \\
a_{2}\left(\mathbf{E}_{2, h}^{n+1}, \mathbf{R}_{2, h} \boldsymbol{\xi}_{h}\right)+Z^{-1} \gamma\left(\mathbf{T r}_{2} \mathbf{E}_{2, h}^{n+1}, \boldsymbol{\xi}_{h}\right)_{X_{\Gamma}} \\
=\int_{\Omega_{2}} \mathbf{F} \cdot \overline{\mathbf{R}_{2, h} \boldsymbol{\xi}_{h}}+\int_{\Omega_{1}} \mathbf{F} \cdot \overline{\mathbf{R}_{1, h} \boldsymbol{\xi}_{h}}-a_{1}\left(\mathbf{E}_{1, h}^{n+1}, \mathbf{R}_{1, h} \boldsymbol{\xi}_{h}\right) \\
+Z^{-1} \gamma\left(\mathbf{T r}_{1} \mathbf{E}_{1, h}^{n+1}, \boldsymbol{\xi}_{h}\right)_{X_{\Gamma}} \quad \forall \boldsymbol{\xi}_{h} \in X_{\Gamma, h},
\end{array}\right.
$$

where the bilinear forms $a_{j}(\cdot, \cdot)$ are the restrictions of the bilinear form $a(\cdot, \cdot)$ to $\Omega_{j}$, and finally set

$$
\boldsymbol{\zeta}_{h}^{n+1}=(1-\theta) \boldsymbol{\zeta}_{h}^{n}+\theta \operatorname{Tr}_{2} \mathbf{E}_{2, h}^{n+1}
$$

Remark 5.2. We can modify algorithm (25-27) substituting in (26) the scalar product in $X_{\Gamma}$ by the scalar product in $L^{2}(\Gamma)$. Since all the norms in a finite dimensional space are equivalent, it is not difficult to prove that also this algorithm converges; however, we can not prove that the rate of convergence of the modified iterative scheme is independent on $h$. 
The $\gamma$-Robin/Robin method for the discrete time-harmonic Maxwell problem reads: being given $\boldsymbol{\zeta}_{h}^{0} \in X_{\Gamma, h}$, for $n \geq 0$ solve for $j=1,2$

$$
\left\{\begin{array}{l}
\mathbf{E}_{j, h}^{n+1} \in V_{j, h}: \\
b_{j}\left(\mathbf{E}_{j, h}^{n+1}, \mathbf{v}_{j, h}\right)=L_{j}\left(\mathbf{v}_{j, h}\right) \quad \forall \mathbf{v}_{j, h} \in V_{j, h}^{0} \\
\operatorname{Tr}_{j} \mathbf{E}_{j, h}^{n+1}=\boldsymbol{\zeta}_{h}^{n}
\end{array}\right.
$$

then

$$
\left\{\begin{array}{l}
\boldsymbol{\Phi}_{j, h}^{n+1} \in V_{j, h}: \\
b_{j}\left(\boldsymbol{\Phi}_{j, h}^{n+1}, \mathbf{v}_{j, h}\right)=0 \quad \forall \mathbf{v}_{j, h} \in V_{j, h}^{0} \\
b_{j}\left(\boldsymbol{\Phi}_{j, h}^{n+1}, \mathbf{R}_{j, h} \boldsymbol{\xi}_{h}\right)+\gamma\left(\operatorname{Tr}_{j} \boldsymbol{\Phi}_{j, h}^{n+1}, \boldsymbol{\xi}_{h}\right)_{X_{\Gamma}}=L_{1}\left(\mathbf{R}_{1, h} \boldsymbol{\xi}_{h}\right) \\
-b_{1}\left(\mathbf{E}_{1, h}^{n+1}, \mathbf{R}_{1, h} \boldsymbol{\xi}_{h}\right)+L_{2}\left(\mathbf{R}_{2, h} \boldsymbol{\xi}_{h}\right)-b_{2}\left(\mathbf{E}_{2, h}^{n+1}, \mathbf{R}_{2, h} \boldsymbol{\xi}_{h}\right) \forall \boldsymbol{\xi}_{h} \in X_{\Gamma, h},
\end{array}\right.
$$

and finally set

$$
\boldsymbol{\zeta}_{h}^{n+1}=\boldsymbol{\zeta}_{h}^{n}+\theta\left(\operatorname{Tr}_{1} \boldsymbol{\Phi}_{1, h}^{n+1}+\operatorname{Tr}_{2} \boldsymbol{\Phi}_{2, h}^{n+1}\right) .
$$

Theorem 5.3. There exist a constant $\gamma^{\sharp} \in \mathbb{R}$ and for each $\gamma \geq 0, \gamma>\gamma^{\sharp}$ there exist an interval $I_{\gamma}=\left(0, \theta_{\gamma}^{0}\right)$ such that for each $\boldsymbol{\zeta}_{h}^{0} \in X_{\Gamma, h}$ and for each $\theta \in I_{\gamma}$ the iterative procedure (28-30) converges. Moreover the rate of convergence is independent of $h$.

Proof. The result is based on Theorem 4.4. In the proof of Theorem 5.1, we have already verified that H1-H3 are satisfied.

As for the $\gamma$-Dirichlet/Robin method, by multiplying (28) and (29) by $Z^{-1}$ we can write the $\gamma$-Robin/Robin method using the original bilinear form $a(\cdot, \cdot)$. Moreover, we can substitute in (29) the scalar product in $X_{\Gamma, h}$ by the scalar product in $L^{2}(\Gamma)$, at the cost of loosing the proof that the rate convergence is independent of $h$.

The modified Neumann/Neumann method for the discrete time-harmonic Maxwell problem reads: being given $\boldsymbol{\zeta}_{h}^{0} \in X_{\Gamma, h}$, for $n \geq 0$ solve for $j=1,2$

$$
\left\{\begin{array}{l}
\mathbf{E}_{j, h}^{n+1} \in V_{j, h}: \\
b_{j}\left(\mathbf{E}_{j, h}^{n+1}, \mathbf{v}_{j, h}\right)=L_{j}\left(\mathbf{v}_{j, h}\right) \quad \forall \mathbf{v}_{j, h} \in V_{j, h}^{0} \\
\operatorname{Tr}_{j} \mathbf{E}_{j, h}^{n+1}=\boldsymbol{\zeta}_{h}^{n}
\end{array}\right.
$$

then

$$
\left\{\begin{array}{l}
\boldsymbol{\Phi}_{j, h}^{n+1} \in V_{j, h}: \\
b_{R, j}\left(\boldsymbol{\Phi}_{j, h}^{n+1}, \mathbf{v}_{j, h}\right)=0 \quad \forall \mathbf{v}_{j, h} \in V_{j, h}^{0} \\
b_{R, j}\left(\boldsymbol{\Phi}_{j, h}^{n+1}, \mathbf{R}_{j, h} \boldsymbol{\xi}_{h}\right)=L_{1}\left(\mathbf{R}_{1, h} \boldsymbol{\xi}_{h}\right)-b_{1}\left(\mathbf{E}_{1, h}^{n+1}, \mathbf{R}_{1, h} \boldsymbol{\xi}_{h}\right) \\
\quad+L_{2}\left(\mathbf{R}_{2, h} \boldsymbol{\xi}_{h}\right)-b_{2}\left(\mathbf{E}_{2, h}^{n+1}, \mathbf{R}_{2, h} \boldsymbol{\xi}_{h}\right) \quad \forall \boldsymbol{\xi}_{h} \in X_{\Gamma, h},
\end{array}\right.
$$


and finally set

$$
\boldsymbol{\zeta}_{h}^{n+1}=\boldsymbol{\zeta}_{h}^{n}+\theta\left(\operatorname{Tr}_{1} \boldsymbol{\Phi}_{1, h}^{n+1}+\operatorname{Tr}_{2} \boldsymbol{\Phi}_{2, h}^{n+1}\right)
$$

By means of Theorem 4.5, the following result can be proved.

Theorem 5.4. There exist $\theta_{0}>0$ such that for each $\theta \in\left(0, \theta_{0}\right)$ the iterative procedure (31-33) converges. Moreover the rate of convergence is independent of $h$.

Finally, we notice that these three domain decomposition methods can be applied to other boundary value problems: for instance, the wave problem in the frequency domain, and the time-harmonic Maxwell equations in a conductor with Robin boundary conditions.

The wave problem in the frequency domain

The problem reads (see [17]):

$$
\left\{\begin{array}{l}
-\Delta u-P u+\mathrm{i} Q u=f \quad \text { on } \Omega \\
\frac{\partial u}{\partial n}-M_{1} u+\mathrm{i} M_{2} u=0 \quad \text { in } \partial \Omega
\end{array}\right.
$$

with $P, Q \in L^{\infty}(\Omega), M_{1}, M_{2} \in L^{\infty}(\partial \Omega)$, and

$$
\begin{aligned}
& Q(\mathbf{x}) \geq Q_{0}>0 \quad \text { for almost all } \mathbf{x} \in \Omega \\
& M_{2}\left(\mathbf{x}^{\prime}\right) \geq M_{2,0}>0 \quad \text { for almost all } \mathbf{x}^{\prime} \in \partial \Omega .
\end{aligned}
$$

Assuming that $f \in L^{2}(\Omega)$, the weak formulation of this problem reads

$$
\left\{\begin{array}{l}
\text { find } u \in H^{1}(\Omega): \\
c(u, v)=g(v) \quad \forall v \in H^{1}(\Omega),
\end{array}\right.
$$

where

and

$$
c(w, v):=\int_{\Omega}(\nabla w \cdot \nabla \bar{v}-P w \bar{v})-\int_{\partial \Omega} M_{1} w \bar{v}+\mathrm{i}\left[\int_{\Omega} Q w \bar{v}+\int_{\partial \Omega} M_{2} w \bar{v}\right]
$$

$$
g(v):=\int_{\Omega} f \bar{v}
$$

The bilinear form $c(\cdot, \cdot)$ is complex valued, continuous and coercive, but it is not Hermitian symmetric and real coercive. However, by multiplying it by an appropriate complex constant we can arrive to an equivalent formulation of problem (34) for which the arguments of Sections 3 and 4 can be repeated.

For the numerical approximation of problem (34) the standard Lagrange finite elements can be used. It is well-known that for this kind of finite elements defined on a regular family of triangulations $\left\{\mathcal{T}_{h}\right\}_{h>0}$ of $\Omega$ which induces a quasi-uniform family of triangulations on $\Gamma$, assumption $\mathrm{H} 3$ of Theorem 4.2 is satisfied with a constant independent of $h$ (see, for instance, $[8,11,19]$ ). Moreover it is clear that assumptions H1 and H2 are satisfied with constants independent of $h$; hence, also for problem (34) we can prove the results reported in Theorems 5.1, 5.3 and 5.4.

The time-harmonic Maxwell equations with damping with Robin boundary conditions

This problems reads (see $[15,16]$ for the case $\sigma=0$ )

$$
\begin{cases}\operatorname{rot}\left(\mu^{-1} \operatorname{rot} \mathbf{U}\right)-\omega^{2} \varepsilon \mathbf{U}+\mathrm{i} \omega \sigma \mathbf{U}=\mathrm{i} \omega \mathbf{J} & \text { on } \Omega \\ \mu^{-1} \operatorname{rot} \mathbf{U} \times \mathbf{n}+\mathrm{i} \omega \mathbf{n} \times(\mathbf{U} \times \mathbf{n})=\mathbf{g} & \text { in } \partial \Omega,\end{cases}
$$


where $\mathbf{J} \in\left(L^{2}(\Omega)\right)^{3}, \mathbf{g} \in\left(L^{2}(\partial \Omega)\right)^{3}$ and $(\mathbf{g} \cdot \mathbf{n})_{\mid \partial \Omega}=0$.

We consider the space

$$
H(\Omega):=\left\{\mathbf{v} \in H(\operatorname{rot} ; \Omega) \mid(\mathbf{v} \times \mathbf{n})_{\mid \partial \Omega} \in\left(L^{2}(\partial \Omega)\right)^{3}\right\},
$$

with the norm

$$
\|\mathbf{v}\|_{H(\Omega)}:=\left(\|\mathbf{v}\|_{L^{2}(\Omega)}^{2}+\|\operatorname{rot} \mathbf{v}\|_{L^{2}(\Omega)}^{2}+\|\mathbf{v} \times \mathbf{n}\|_{L^{2}(\partial \Omega)}^{2}\right)^{1 / 2} .
$$

The weak formulation of this problem reads

$$
\left\{\begin{array}{l}
\text { find } \mathbf{U} \in H(\Omega): \\
d(\mathbf{U}, \mathbf{v})=G(\mathbf{v}) \quad \forall \mathbf{v} \in H(\Omega),
\end{array}\right.
$$

where

$$
d(\mathbf{w}, \mathbf{v}):=a(\mathbf{w}, \mathbf{v})+\mathrm{i} \omega \int_{\partial \Omega}(\mathbf{w} \times \mathbf{n}) \cdot(\overline{\mathbf{v}} \times \mathbf{n}),
$$

$a(\cdot, \cdot)$ is defined in $(4)$, and

$$
G(\mathbf{v}):=\mathrm{i} \omega \int_{\Omega} \mathbf{J} \cdot \overline{\mathbf{v}}+\int_{\partial \Omega}(\mathbf{g} \times \mathbf{n}) \cdot(\overline{\mathbf{v}} \times \mathbf{n}) .
$$

The bilinear form $d(\cdot, \cdot)$ is complex valued, continuous and coercive, but it is not Hermitian symmetric and real coercive. Then we need to multiply it by an appropriate complex constant in order to repeat the arguments of Sections 3 and 4.

With the technical assumption $\Gamma \cap \partial \Omega=\emptyset$ it is easy to see that, using the same finite elements employed for problem (8), Theorems 5.1, 5.3 and 5.4 still hold for problem (35).

\section{Numerical tests}

In this Section we present some numerical tests illustrating the performances of the algorithms we have proposed. We consider very simple geometric situations, with the only aim of verifying that the convergence of the domain decomposition schemes is achieved, uniformly with respect to the mesh size $h$.

In particular, we have considered the simplest algorithms, always choosing the parameter $\gamma$ equal to 0: though this situation is not covered by the theory presented here, this choice is the easiest to implement and reduces the $\gamma$-Dirichlet/Robin and the $\gamma$-Robin/Robin iterative schemes to the well-known Dirichlet/Neumann and Neumann/Neumann ones, respectively. Numerical evidence will show that both the Dirichlet/Neumann and the Neumann/Neumann schemes are indeed convergent, with a rate independent of the mesh size, and the number of iterations is in general extremely small (provided that the acceleration parameter $\theta$ is properly chosen). Consequently, it is apparent that other choices of the parameter $\gamma$ are not necessary in numerical computations.

The computational domain is always the parallelepiped $\Omega=(0,2) \times(0,1) \times(0,1)$, which will be decomposed into two subdomains $\Omega_{1}=\left(0, x_{\Gamma}\right) \times(0,1) \times(0,1)$ and $\left.\Omega_{2}=\left(x_{\Gamma}, 2\right)\right) \times(0,1) \times(0,1)$. The numerical mesh is uniform, and each element of the grid is a cube of side $h$. We employ the edge elements of Nédélec type (see [21]), with 12 degrees of freedom for each element, one for each edge.

We consider three types of model problems: the first and most important one is the boundary value problem

$$
\begin{cases}\operatorname{rot} \operatorname{rot} \mathbf{u}+\omega(\mathrm{i} \sigma-\omega) \mathbf{u}=\mathbf{f} & \text { in } \Omega \\ \mathbf{u} \times \mathbf{n}=\mathbf{g} & \text { on } \partial \Omega,\end{cases}
$$

which is the complete problem (2), (3) (taking, for simplicity, $\mu=1, \varepsilon=1$ ). 
The second model problem is

$$
\begin{cases}\operatorname{rot} \operatorname{rot} \mathbf{u}+\mathrm{i} \mathbf{u}=\mathbf{f} & \text { in } \Omega \\ \mathbf{u} \times \mathbf{n}=\mathbf{g} & \text { on } \partial \Omega\end{cases}
$$

which is the low-frequency problem analysed in [3] (here we have taken, for simplicity, $\mu=1, \sigma=1$ and $\omega=1$ ). In [3] we have proved that in this case the convergence of the Dirichlet/Neumann scheme is achieved, uniformly with respect to the mesh size $h$.

The third model problem is

$$
\begin{cases}\operatorname{rot} \operatorname{rot} \mathbf{u}+\mathbf{u}=\mathbf{f} & \text { in } \Omega \\ \mathbf{u} \times \mathbf{n}=\mathbf{g} & \text { on } \partial \Omega\end{cases}
$$

This is the best possible test case for the Dirichlet/Neumann iterative scheme for rot-conforming finite elements, as the weak form associated to the operator rot rot $+I$ is the scalar product in $H$ (rot; $\Omega$ ), and therefore the problem is hermitian and coercive. In this case, the theoretical results assure that the Dirichlet/Neumann scheme is convergent, uniformly in $h$ (see [23], Chap. 4).

For the numerical computations we used the standard tool-box sparfun of MATLAB ${ }^{\mathrm{TM}}$ 5.2. In particular, for solving the linear systems we adopt the gmres function, with $T O L=10^{-6}$.

In the iteration-by-subdomain procedure, we have used the following stopping test

$$
\sum_{i=1}^{2} \frac{\left\|\mathbf{u}_{i, h}^{k+1}-\mathbf{u}_{i, h}^{k}\right\|_{H\left(\operatorname{rot} ; \Omega_{i}\right)}^{2}}{\left\|\mathbf{u}_{i, h}^{k+1}\right\|_{H\left(\operatorname{rot} ; \Omega_{i}\right)}^{2}} \leq 10^{-4}
$$

The first numerical test concerns the exact solution $\mathbf{u}(x, y, z)=\left(z^{2}, x^{2}, y^{2}\right)$ (having computed consequently the data $\mathbf{f}$ and $\mathbf{g}$ ). We have considered problems (36), (37) and (38) (the first one with $\sigma=1$ and $\omega=1$ ), with different values of $h$ (corresponding to the degrees of freedom indicated in Table 6.1).

TABLE 6.1. Choice of the mesh size $h$

(the correspondent degrees of freedom are also indicated).

\begin{tabular}{|c|cccccc|}
\hline$h$ & $1 / 4$ & $1 / 6$ & $1 / 8$ & $1 / 10$ & $1 / 12$ & $1 / 14$ \\
DOF & 240 & 960 & 2464 & 5040 & 8976 & 14560 \\
\hline
\end{tabular}

Choosing $x_{\Gamma}=0.5, \theta=0.5$, the number of iterations of the 0 -DR (DN) method has been always equal to 4 . We have repeated the computations for two other exact solutions $\left(\mathbf{u}(x, y, z)=\left(\sin z, \cos x, \mathrm{e}^{y}\right), \mathbf{u}(x, y, z)=\right.$ $\left.\left(\mathrm{e}^{z} \sin (x y),(y+z) \mathrm{e}^{x}, \cos (x z)\right)\right)$, for all problems (36), (37) and (38) (the first one with $\sigma=1$ and $\left.\omega=1\right)$, and $h$ as in Table 6.1. For the set of problems with the exact solution $\mathbf{u}(x, y, z)=\left(\sin z, \cos x, \mathrm{e}^{y}\right)$ we have taken $x_{\Gamma}=0.5$, and for the set of problems with the exact solution $\mathbf{u}(x, y, z)=\left(\mathrm{e}^{z} \sin (x y),(y+z) \mathrm{e}^{x}, \cos (x z)\right)$ we have taken $x_{\Gamma}=1.5$. Choosing as before $\theta=0.5$, the convergence results have been quite similar (always 4 iterations to achieve convergence).

It is worthy of noting that the best choice of the relaxation parameter $\theta$ seems to be always very close to 0.5 . The next computations are made for the exact solution $\mathbf{u}(x, y, z)=\left(z^{2}, x^{2}, y^{2}\right)$ of problem (36) (again with $\sigma=1$ and $\omega=1$ ), having chosen $x_{\Gamma}=4 / 3$. 
TABLE 6.2. Number of iterations for the 0 -DR (DN) method for different values of $\theta$ and $h$.

\begin{tabular}{|r|r|r|r|}
\hline$\theta \backslash h(D O F)$ & $1 / 3(84)$ & $1 / 6(960)$ & $1 / 9(3600)$ \\
\hline 0.4 & 5 & 6 & 6 \\
0.45 & 5 & 5 & 5 \\
0.5 & 4 & 4 & 4 \\
0.55 & 4 & 5 & 5 \\
0.6 & 5 & 6 & 6 \\
\hline
\end{tabular}

For the solution $\mathbf{u}(x, y, z)=\left(\sin z, \cos x, \mathrm{e}^{y}\right)$, taking $h=1 / 6(D O F=960)$, the best choice of $\theta$ is reported in Tables 6.3 and 6.4. Note that only choosing $\theta$ very close to 0.5 in problem (38) we have been able to obtain a better rate of convergence than in the case $\theta=0.5$.

TABLE 6.3. Number of iterations for the 0-DR (DN) method for different values of $\theta$ and $x_{\Gamma}$ (problem (36)).

\begin{tabular}{|r|r|r|}
\hline$\theta \backslash x_{\Gamma}$ & $4 / 3$ & $2 / 3$ \\
\hline 0.3 & 8 & 7 \\
0.4 & 6 & 5 \\
0.5 & 4 & 4 \\
0.6 & 6 & 6 \\
0.7 & 8 & 9 \\
\hline
\end{tabular}

TABLE 6.4. Number of iterations for the 0-DR (DN) method for different values of $\theta$ and $x_{\Gamma}$ (problem (38)).

\begin{tabular}{|r|r|r|}
\hline$\theta \backslash x_{\Gamma}$ & $4 / 3$ & $2 / 3$ \\
\hline 0.3 & 7 & 7 \\
0.4 & 5 & 5 \\
0.495 & 4 & 3 \\
0.5 & 4 & 4 \\
0.504 & 3 & 4 \\
0.6 & 6 & 6 \\
0.7 & 8 & 8 \\
\hline
\end{tabular}

These results lead to a significant conclusion: in all cases we have analysed, the choice of relaxation parameter $\theta$ equal to 0.5 can be considered optimal for the Dirichlet/Neumann method. Moreover, the method is quite efficient also for a wide range of values of $\theta$.

We have also verified that the 0-Robin/Robin scheme (which indeed is coincident with the classical Neumann/Neumann scheme) is extremely efficient. In fact, we have considered the same exact solutions than before for problem (36) (with $\sigma=1$ and $\omega=1$ ), choosing $x_{\Gamma}=1.5, \theta=0.25$ (which seems to be optimal for the Neumann/Neumann method), and the values of $h$ indicated in Table 6.1. In all these cases, the number of iterations has been always equal to 3 .

The results for the modified Neumann/Neumann iterative scheme are less interesting. We have considered the exact solutions $\mathbf{u}(x, y, z)=\left(\sin z, \cos x, \mathrm{e}^{y}\right)$ and $\mathbf{u}(x, y, z)=\left(\mathrm{e}^{z} \sin (x y),(y+z) \mathrm{e}^{x}, \cos (x z)\right)$ for problem (36) (with $\sigma=1$ and $\omega=1$ ). Choosing $x_{\Gamma}=1$, the convergence results are reported in Tables 6.5 and 6.6. 
TABLE 6.5. Number of iterations for the modified Neumann/Neumann method for different values of $\theta$ and $h\left(\mathbf{u}(x, y, z)=\left(\sin z, \cos x, \mathrm{e}^{y}\right)\right)$.

\begin{tabular}{|c|r|r|r|}
\hline$\theta \backslash h$ & $1 / 4$ & $1 / 6$ & $1 / 8$ \\
\hline 0.05 & 33 & 35 & 37 \\
0.10 & NO & NO & NO \\
0.25 & NO & NO & NO \\
\hline
\end{tabular}

TABLE 6.6. Number of iterations for the modified Neumann/Neumann method for different values of $\theta$ and $h\left(\mathbf{u}(x, y, z)=\left(\mathrm{e}^{z} \sin (x y),(y+z) \mathrm{e}^{x}, \cos (x z)\right)\right)$.

\begin{tabular}{|c|c|c|c|}
\hline$\theta \backslash h$ & $1 / 4$ & $1 / 6$ & $1 / 8$ \\
\hline 0.05 & 279 & 276 & 276 \\
0.10 & NO & NO & NO \\
0.25 & NO & NO & NO \\
\hline
\end{tabular}

One can see that, as predicted by the theory, the scheme is indeed convergent for a suitable (interval of) $\theta$. However, since this value is rather small, convergence is quite slow. The reason is that the preconditioner is relatively different from the given operator (the latter is not hermitian nor real coercive, the former is real, symmetric and coercive). As a consequence, its preconditioning properties are poor (though the rate of convergence is in fact independent of $h$ ).

In conclusion, the computations performed show that both the Dirichlet/Neumann and Neumann/Neumann iterative schemes, though not covered by the theory, are extremely efficient and robust domain decomposition methods for the time-harmonic Maxwell equations with damping.

\section{REFERENCES}

[1] V.I. Agoshkov and V.I. Lebedev, Poincaré-Steklov operators and the methods of partition of the domain in variational problems, in Vychisl. Protsessy Sist. (Computational processes and systems), G.I. Marchuk, Ed., Nauka, Moscow 2 (1985) 173-227 (in Russian).

[2] A. Alonso and A. Valli, Some remarks on the characterization of the space of tangential traces of $H$ (rot; $\Omega$ ) and the construction of an extension operator. Manuscripta Math. 89 (1996) 159-178.

[3] A. Alonso and A. Valli, An optimal domain decomposition preconditioner for low-frequency time-harmonic Maxwell equations. Math. Comp. 68 (1999) 607-631.

[4] A. Alonso and A. Valli, A domain decomposition approach for heterogeneous time-harmonic Maxwell equations. Comput. Methods Appl. Mech. Engrg. 143 (1997) 97-112.

[5] A. Alonso, R.L. Trotta and A. Valli, Coercive domain decomposition algorithms for advection-diffusion equations and systems. J. Comput. Appl. Math. 96 (1998) 51-76.

[6] L.C. Berselli, Some topics in fluid mechanics. Ph.D. thesis, Dipartimento di Matematica, Università di Pisa, Italy (1999).

[7] L.C. Berselli and F. Saleri, New substructuring domain decomposition methods for advection-diffusion equations. J. Comput. Appl. Math. 116 (2000) 201-220.

[8] P.E. Bjørstad and O.B. Widlund, Iterative methods for the solution of elliptic problems on regions partitioned into substructures. SIAM J. Numer. Anal. 23 (1986) 1097-1120.

[9] A. Bossavit, Électromagnétisme, en vue de la modélisation. Springer-Verlag, Paris (1993).

[10] J.-F. Bourgat, R. Glowinski, P. Le Tallec and M. Vidrascu, Variational formulation and algorithm for trace operator in domain decomposition calculations, in Domain Decomposition Methods, T.F. Chan et al., Eds., SIAM, Philadelphia (1989) 3-16.

[11] J.H. Bramble, J.E. Pasciak and A.H. Schatz, An iterative method for elliptic problems on regions partitioned into substructures. Math. Comp. 46 (1986) 361-369.

[12] A. Buffa and P. Ciarlet, Jr., On traces for functional spaces related to Maxwell's equations Part I: An integration by parts formula in Lipschitz polyhedra. Math. Methods Appl. Sci. 24 (2001) 9-30.

[13] A. Buffa and P. Ciarlet, Jr., On traces for functional spaces related to Maxwell's equations Part II: Hodge decompositions on the boundary of Lipschitz polyhedra and applications. Math. Meth. Appl. Sci. 24 (2001) 31-48. 
[14] M. Cessenat, Mathematical methods in electromagnetism: Linear theory and applications. World Scientific Pub. Co., Singapore (1996).

[15] P. Collino, G. Delbue, P. Joly and A. Piacentini, A new interface condition in the non-overlapping domain decomposition method for the Maxwell equation. Comput. Methods Appl. Mech. Engrg. 148 (1997) 195-207.

[16] B. Després, P. Joly and J.E. Roberts, A domain decomposition method for the harmonic Maxwell equation, in Iterative Methods in Linear Algebra, R. Beaurvens and P. de Groen, Eds., North Holland, Amsterdam (1992) 475-484.

[17] S. Kim, Domain decomposition iterative procedures for solving scalar waves in the frequency domain. Numer. Math. 79 (1998) 231-259.

[18] R. Leis, Exterior boundary-value problems in mathematical physics, in Trends in Applications of Pure Mathematics to Mechanics 11, H. Zorski, Ed., Pitman, London (1979) 187-203.

[19] L.D. Marini and A. Quarteroni, A relaxation procedure for domain decomposition methods using finite elements. Numer. Math. 55 (1989) 575-598.

[20] P. Monk, A finite element method for approximating the time-harmonic Maxwell equations. Numer. Math. 63 (1992) $243-261$.

[21] J.C. Nédélec, Mixed finite elements in $\mathbb{R}^{3}$. Numer. Math. 35 (1980) 315-341.

[22] J.C. Nédélec, A new family of mixed finite elements in $\mathbb{R}^{3}$. Numer. Math. 50 (1986) 57-81.

[23] A. Quarteroni and A. Valli, Domain decomposition methods for partial differential equations. Oxford University Press, Oxford (1999).

[24] J.E. Santos, Global and domain-decomposed mixed methods for the solution of Maxwell's equations with application to magnetotellurics. Numer. Methods. Partial Differ. Equations 14 (1998) 407-437.

[25] A. Toselli, Domain decomposition methods for vector field problems. Ph.D. thesis, Courant Institute, New York University, New York (1999).

To access this journal online:

www.edpsciences.org 